\title{
Application of Texture Analysis technique in formulation development of lyophilized orally disintegrating tablets containing mannitol, polyvinylpyrrolidone and amino acids
}

\author{
Ellen Hackl ${ }^{\&}$, Irina Ermolina*
}

Leicester School of Pharmacy, Faculty of Health and Life Sciences, De Montfort University, Leicester, UK

\& present address: School of Pharmacy, University of Reading, UK

*corresponding author, iermolina@dmu.ac.uk

Keywords: orally disintegrating tablets, freeze-drying, texture analysis, PVP, amino acids

\begin{abstract}
Abbreviation:
ODTs - orally disintegrating tablets, RDTs - rapid disintegrating tablets, AA - amino acid, PVP Polyvinylpyrrolidone, TA - Texture Analysis, FD - freeze-drying / freeze-dried, RH - relative humidity, DSC Differential Scanning Calorimetry, SEM - Scanning Electron Microscopy, CQA - Critical Quality Attributes
\end{abstract}

\section{Running head:}

Application of Texture Analysis in formulation development of lyophilized ODTs

\begin{abstract}
Orally disintegrating tablets (ODTs) attract a great attention as this easy swallowing dosage form often improves the patient compliance. In the current work orally disintegrating tablets comprising mannitol, polyvinylpyrrolidone and an amino acid (alanine, glycine or serine) with various PVP-to-amino acid ratios were formulated. The combination of mannitol and an amino acid was aimed to use the advantages of mannitol, the matrix supporting and disintegration agent, and to reduce the total amount of sugar/polyol in tablets. Tablets were manufactured by freeze-drying and their properties (appearance, internal structure, disintegration, mechanical and texture properties, moisture uptake, shrinkage, thermal properties) were assessed. In the work great emphasis was placed on illustrating the applicability of the Texture Analysis of the freeze-dried cakes directly in vials in formulation development.

The results show that appearance, the mechanical properties, disintegration and shrinkage of the freezedried ODTs depend significantly on the excipient composition with PVP playing the leading role. Partial mannitol replacement with an amino acid has a limited impact on the tablet properties. The presence of an amino acid also has no impact on the PVP-mannitol interaction. The mechanical and texture properties of freeze-dried ODTs depend non-linearly on the PVP content. The transition between the different types of textures occurs in a narrow interval of PVP concentrations regardless of the type of amino acid in a formulation. The non-linear effect of PVP on various tablet properties should be taken into account when designing ODT formulations as it can compromise the robustness of the manufacturing process.
\end{abstract}




\section{Introduction}

Orally (or rapid) disintegrating tablets (ODTs or RDTs) attract a great deal of attention as this easy swallowing dosage form often improves the patient compliance $[1,2,3,4,5,6]$. ODTs combine the advantages of solid dosage forms (accurate dosing, easy handling and small package size) with the advantages of liquid formulations (easy administration). Dysphagia, or difficulty in swallowing, is common in about 35\% of the general population [7] especially among pediatric and geriatric patients as well as institutionalized and psychiatric patients. Recent survey of healthcare professionals working across two hospitals named ODTs as the second most popular pediatric dosage form (after liquids) and 63.0\% of practitioners agreed that many liquid formulations could be substituted with a suitable ODT [8]. In addition, naloxone orally disintegrating tablets were recently tested for using in opioid overdose treatment (naloxone, an accepted opioid antagonist used to block the effects of opioids, especially in overdose, is shown to be absorbed via the buccal route [9]). In this case orally disintegrating tablets offer a convenient way of drug administration for first responders (non-medical personal unable to carry out injectable treatments). In the last decade rapid disintegrating tablets also became an attractive option for formulating oral vaccines since this dosage form packaged in a compact stackable blister pack can be easily distributed and administered directly to patients without reconstitution [10]. In particular, recently it was shown that freeze-dried orally disintegrating tablets entrapping antigen-containing bilayer vesicles (which released upon rehydration) are suitable for the oral delivery of vaccines [11].

According to recent assessment carried out by Persistence Market Research 'Orally Disintegrating Tablets Market: Global Industry Analysis 2012 - 2016 and Forecast 2017 - 2025', the global market revenue for ODTs is expected to increase significantly [12]. Anti-psychotics drug class was the dominant drug class segment (followed by anti-epileptics, CNS stimulants, anxiolytics, and anti-Parkinsonian drugs) in the global ODTs market in 2016 and this segment is expected to further gain traction.

Freeze-drying, or lyophilisation, is one of the main techniques in ODTs production leading in the sales volume and number of products on the market [13, 14 and references 1-4 therein, 15, 16, 17]. ODTs prepared by lyophilisation are very light and possesses highly porous structure that guarantees rapid disintegration/dissolution in the mouth without chewing and the need for water; however, it reduces mechanical strength and storage stability of the tablets [18]. The main desirable critical quality attributes for the successful ODTs include (i) fast disintegration with minimum amount of liquid (saliva) and (ii) a sufficient mechanical strength to withstand the transportation, storage and handling without fragility concern $[3,19$, 20]. Therefore, in addition to disintegration rate, the mechanical properties of the ODTs need to be studied to ensure the development of a successful ODT formulation.

In our recent work [21] we have suggested the application of the standard Texture Analysis method to assess the texture and mechanical properties of the freeze-dried cakes directly in the glass vials. The approach proposed allows simple and quick evaluation of the mechanical properties and the texture of the dry cakes and cakes underwent different treatments. The main advantage of the approach is the ability to robustly assess an intact cake without necessity to transfer it from a vial or cut (or disrupt in any other way) a FD sample in order to make a measurement. The aim of the present work was to illustrate the applicability of the texture analysis assessment in vials in formulation development for orally disintegrating tablets. 
The most well-known example of the freeze-drying method for ODTs production is the Zydis ${ }^{\circledR}$ technology involving drug physical trapping in a matrix composed of two components, a water soluble polymeric structure former (a binder) and a saccharide/polyol (typically mannitol) [22 and references 10-12 therein, 23]. The former maintains the shape and provides the mechanical strength while the latter serves as a matrix supporting and disintegration accelerating agent which cements the porous polymer framework and enhances disintegration $[20,23,24$,$] .$

The number of matrix-supporting agents used has been mainly limited to saccharides and polyols. Mannitol is the most commonly used polyol in the ODT formulations [24,25]. It has many benefits as a low hygroscopic, stable during FD and inert towards API excipient with good compactibility and the tendency to crystallize during lyophilisation. Mannitol has a very low glycemic index and is considered to be insulinneutral (i.e. not raising blood glucose and insulin levels) [26]. However, mannitol as well as other sugar alcohols is a low digestible carbohydrate and only partially absorbed from the small intestine. Colonic bacteria in the lower part of the intestinal tract can metabolize some of the non-absorbed mannitol that might cause upset stomach, more intestinal gas, bloating or diarrhea. Besides, mannitol can also acts as an osmotic diuretic, thus reducing blood pressure. A patient's response to mannitol side effects will depend on individual factors as well as the general amount and frequency of consumption. The U.S. FDA's regulation for mannitol requires label statement "Excess consumption may have a laxative effect" if food consumption may excess 20 grams of mannitol per day.

It is known that ODTs are often used for treatment of long-term chronic conditions such as depression, anxiety, mood disorders, insomnia (ODTs account for more than $40 \%$ of the market value of therapies for central nervous system disorder), gastrointestinal problems, diabetes, cancer [12, 27, 28,]. Long-lasting treatment, as well as treatment for children, diabetic and obese patients require limited intake of saccharides and polyols. Therefore the general tendency in the current drug formulation development is to replace saccharides with alternative excipients, for instance, amino acids (AA). The feasibility of using different amino acids as matrix supporting agents in rapid disintegrating tablets was studied in [14]. Authors have shown that eleven (except proline) out of twelve amino acids studied (alanine, arginine, threonine, glycine, cysteine, serine, histidine, lysine, valine, asparagine, glutamine and proline) are suitable for the lyophilisation and ODT production.

In the present work we aimed to combine both mannitol and amino acids in the formulations. This combination allows using the advantages of mannitol (which provides an elegant cake appearance and improved texture, rigidity and nice mouthfeel) and, at the same time, reduces the total amount of sugar/polyol in tablets. In particular, we aimed to study the effect of mannitol replacement with several amino acids on the various properties of ODTs.

One of the parameters that need to be accounted for when choosing an amino acid is a taste of amino acids. It is known that some amino acids have a sweet taste (for example, glycine, alanine, proline, serine, threonine) while some possess a bitter (phenylalanine, tyrosine, leucine, valine, histidine, arginine) or Umami and sour (glutamate, aspartic acid) taste [29]. The presence of bitter amino acids in an oral formulation might require additional sweeteners, whereas sweet amino acids, even having the sweetness lighter than that of sugar, can be used without taste-masking excipients. Therefore alanine, glycine and 
serine were selected for the screening process. These amino acids, together with sweet taste, were shown to increase the stability of formulations comprising AA and gelatin [14].

Polyvinylpyrrolidone (PVP), one of the most widely used polymers in medicine, has been chosen as a binding polymer as it is soluble in water, and has very low cytotoxicity and excellent biocompatibility with living tissues [30]. Besides, PVP unlike some gelatins, is suitable for vegans.

\section{Materials and Methods}

\section{Materials}

D-mannitol, Polyvinylpyrrolidone 30K (PVP30K hereinafter also referred to as PVP for simplicity), L-alanine (Ala), L-glycine (Gly), L-serine (Ser), L-proline (Pro) were purchased from Sigma-Aldrich Co, UK. Unless otherwise specified, all materials and reagents used were of analytical grade and were used for analysis without further purification.

\section{Sample preparation}

All stock solutions (with 5 or $10 \mathrm{wt} \%$ of solid material) were prepared using double-distilled water and mixed directly prior to freeze-drying. In all sets of 3-excipient samples, mannitol content was kept constant (20 or 40 wt\%) while PVP30K and amino acid concentrations were varied with 10 wt\% step (Table 1). Each formulation included only one type of amino acid.

For comparison, two-excipient formulations containing various proportions of PVP and either mannitol or an amino acid (Ala, Gly or Ser) (from PVP:Man(AA)=90:10 to 10:90 with $10 \mathrm{wt} \%$ step) were prepared (Table 1).

All samples were freeze-dried in $10 \mathrm{~mL}$ transparent SCHOTT glass vials (Adelphi Healthcare Packaging, UK) with $2 \mathrm{~mL}$ of solution per vial using FD cycle described below.

\section{Freeze-Drying}

Freeze-dried cakes were prepared using a laboratory scale Smart Lyo2 Freeze-dryer (GEA Lyophil, Germany). $10 \mathrm{ml}$ vials containing $2 \mathrm{ml}$ of solution each were places in the freeze-dryer and kept frozen at $-35{ }^{\circ} \mathrm{C}$ for 6 hours. The primary drying was performed at $-33^{\circ} \mathrm{C}$ for 40 hours with the condenser temperature set at -70 ${ }^{\circ} \mathrm{C}$ and the pressure inside the freeze-dryer chamber at $\sim 100 \mu$ Bar. Then the shelf temperature was increased from -33 to $+20{ }^{\circ} \mathrm{C}$ over a period of 6 hours and the secondary drying was performed at these temperature and pressure for 10 hours. After the freeze-drying cycle has been completed, the vials were fully stoppered under vacuum conditions and kept in a dry atmosphere desiccator to minimize the water vapour sorption into the FD cakes.

\section{Texture Analysis}

The mechanical properties of the freeze-dried cakes were studied by using the Texture Analyser (TA.XT PLUS, Stable Micro Systems, UK) in the compression mode ("hold until time' program with $20 \mathrm{~s}$ "hold on" time). FD cakes were analysed directly in glass vials used for freeze-drying as we described previously in [21]. To penetrate a cake, a stainless steel cylindrical $\mathrm{P} / 6$ probe with torsion area $20.27 \mathrm{~mm}^{2}$ was applied. The penetration depth (distance option) was $3 \mathrm{~mm}$, the speed of penetration (test speed) was $0.01 \mathrm{~mm} / \mathrm{s}$, pretest speed was $1 \mathrm{~mm} / \mathrm{s}$, and the trigger force was chosen as $1 \mathrm{~g}$. 
Stress (the force per unit area) values were calculated according to the following formula

Stress $\left(\frac{g}{m m^{2}}\right)=\frac{\text { Force }(g)}{\text { Probe area }\left(m m^{2}\right)}$

Stress relaxation was calculated as a ratio between the values of force before and after the "on hold" time.

The following parameters were calculated using TA Exponent software (http://www.stablemicrosystems.com): the numbers of positive peaks, linear distance (i.e. the length of a line which join all points measured), average gradient of all positive slopes, and average drop off in force between two consecutive peaks (unless otherwise specified, $50 \mathrm{~g}$ threshold force value was used in calculations). All measurements were done in triplicate and the average values were taken.

\section{Differential Scanning Calorimetry (DSC)}

DSC melting thermograms were recorded using a Perkin-Elmer Jade DSC (USA). Lyophilized powder (5 - 10 $\mathrm{mg}$ ) was crimped in an aluminum DSC pan with vented lids and an experiment was started immediately. Samples were heated from $-60^{\circ} \mathrm{C}$ to $315^{\circ} \mathrm{C}$ at $20^{\circ} \mathrm{C} / \mathrm{min}$. Nitrogen was used as a purge gas.

\section{In vitro disintegration test}

$1 \mathrm{~mL}$ of double-distilled water containing malachite green dye (for contrast and better observation) was added to a glass vial containing freeze-dried cake and the time taken for complete disintegration of the cake (with no visible solid particles remaining) was measured.

\section{Moisture sorption}

Freeze-dried formulations in glass vials or in aluminium boats were incubated at room temperature and at increased relative humidity $(\mathrm{RH})(75 \%$ and $97 \% \mathrm{RH})$ in desiccators with $\mathrm{NaCl}$ and $\mathrm{K}_{2} \mathrm{PO}_{4}$ saturated solutions, respectively (distilled water and chemically pure salts were used). For the total moisture uptake the samples were moisturised for 7-10 days following by 20-hours drying in the oven at 95 oc (temperature was gradually raised from 50 to $95 \stackrel{\circ}{\circ}$ during two hours). Weights of the samples in wet and dried states were measured gravimetrically and moisture uptakes were calculated as $\left(W_{1}-W_{2}\right) / W_{1} * 100 \%$, where $W_{1}$ is weight of moisturised sample, $W_{2}$ is weight of dry sample.

\section{Shrinkage of the freeze-dried cakes}

The FD cakes in glass transparent vials were incubated at $75 \% \mathrm{RH}$ in a desiccator containing saturated $\mathrm{NaCl}$ solution. At defined time intervals, the bottoms of vials were scanned using a conventional scanner and the cake diameters were measured using a Vernier caliper. Each measurement was done in triplicate and an average value was calculated. All diameter values were normalised using the diameter of a freshly prepared dry freeze-dried cake of the same formulation as a reference. A decrease in the cake diameter has been ascribed to the cake shrinkage.

\section{Scanning Electron Microscopy (SEM)}

Internal structures of the lyophilized samples were visualized using a Carl Zeiss EVO HD scanning electron microscope (UK). Thin vertical cross-section of a cake was cut from the lyophilized sample, placed on 12.5 
SEM aluminum stubs using $12 \mathrm{~mm}$ carbon tabs (Agar scientific) and plated with a thin layer of gold using a sputter cutter in a low-pressure argon atmosphere.

\section{Results}

\section{Physical appearance of the FD cakes}

All 2- and 3-excipient formulations studied formed uniform white well-shaped freeze-dried cakes without evidences of collapse (representative examples of the 3-excipient cakes are shown in Fig. 1). Freeze-dried cakes occupied the same volume as the liquid that was filled into the vials. Cakes comprising with low PVP content were better adhered to vials, whilst PVP-rich cakes (PVP > $60 \mathrm{wt} \%$ ) were worse adhered with a number of small cracks at the edges. Mannitol replacement with the amino acids had almost no effect on the appearance of the FD cakes; however, Gly-containing cakes revealed slightly less adherence to walls than Ala- and Ser-containing cakes. Cakes containing a small amount of mannitol (5-20 wt\%) in the 3excipient formulations were mainly better shaped and adhered to the vial walls than those containing no mannitol (2-excipient formulations).

The surfaces of the cakes formed by 2-excipient formulations (mannitol-AA and PVP-AA) were rough with a number of small "bumps". Generally, it is considered that the CQAs are not impacted by the presence of these imperfections in lyophilised products [31]; however, in the case of ODT products with non-flat "bumpy" surface could be less acceptable to the users.

\section{Internal structures}

To compare the morphology of PVP-rich and PVP-deficient solid formulations, the internal structures of the lyophilized cakes comprising mannitol (20\%), PVP30K and Ser were visualized using SEM. As is seen from Fig. 2, freeze-dried formulations with different PVP contents show alike regular closed-cell hexagonal structures with comparable cell dimensions and cell wall thicknesses. Similar hexagonal structures for the different freeze-dried excipients (pure mannitol, gelatin and maltodextrin) were reported previously by us [21] as well as by other researchers (for instance, mannitol, sucrose and trehalose structures are presented in [32, 33]). Regardless of the PVP content, the internal structures of the FD cakes reflect the geometrical structure of the ice crystals forming during the freezing stage.

\section{Disintegration}

All the 2- and 3-excipient cakes studied had the disintegration times of less than $30 \mathrm{~s}$. The disintegration times for AA-rich formulations with PVP30K content below $30 \mathrm{wt} \%$ were in the 5-15 second interval while the disintegration times for the PVP-rich formulations were within 20-30 s regardless of the amino acid type. Increasing the mannitol content from 20 to $40 \mathrm{wt} \%$ reduced the disintegration times for PVP-rich formulations to $15-20 \mathrm{~s}$. The disintegration times were shown to be dependent mostly on the PVP30K content rather than on the type of the amino acid.

\section{Moisture uptake and tablet shrinkage}

Moisture uptake by the 3-excipient ODTs comprising mannitol, PVP30K and an amino acid (Ala, Gly or Ser) was studied by incubating the open glass vials at high moisture $(75 \% \mathrm{RH}$, room temperature) over time. Moisture-induced cake shrinkage was assessed by scanning the vials and calculating the decrease in the cake average diameter. A representative scan is shown in Fig. 3 (a); Fig. 3 (b) presents the representative 
dependencies of the relative diameters of FD cakes on time for ODTs containing glycine. The shrinkage results (Fig. 3) show that FD cakes with low and intermediate PVP content $(<50 \%)$ preserve their shape at elevated relative humidity for a relatively long time. High PVP content $(>60 \%)$ in cakes leads to a significant cake shrinkage in the first 24 hours of moisture treatment (Fig. 3 (b) and (c)). On average, the degree of shrinkage is higher for the cakes containing Gly or Ser than for the cakes containing Ala. The results on the FD cake shrinkage (Fig. 3) also show that for some formulations the dependence of shrinkage on the PVP content is non-monotonous.

Following the incubation at high humidity, the total moisture uptake by FD cakes was assessed. As Fig. 4 shows, increase in the PVP concentration and, consequently, decrease in the amino acid concentration (with a fixed amount of mannitol in the formulations) leads to a significant increase in the moisture content in the freeze-dried cakes. Comparison of the total moisture uptake by formulations containing different amino acids reveals that the PVP content is primary responsible for the amount of moisture accumulated (at constant concentration of mannitol).

The moisture treatment of the freeze-dried cakes in vials at $75 \% \mathrm{RH}$ could mimic the conditions of a very limited amount of liquid (saliva) in the mouth. Thus it is of interest to examine the state of the moisturized tablets and how easily they can be dissolved/disintegrated if a small amount of water ( $5 \mathrm{ml})$ is added. Moisturized tablets with low (0-20 wt\%) and low-to-average (20-40 wt\%) PVP contents were similar to soft wet powder and soft non-stick moist cotton, respectively. They dissolved very fast (in less than $5 \mathrm{sec}$ ) upon addition of water. In contrast, tablets with high PVP content (above $70 \mathrm{wt} \%$ ) and, consequently, low to zero amino acid content became similar to hard rubber (Ala- and Gly-containing tablets) or toffee (Ser-containing tablets) after the moisture treatment. The PVP-rich moisturized tablets also became very sticky and required long time ( $1 \mathrm{~min}$ for Ser tablets and 2-3 $\mathrm{min}$ for Ala- and Gly tablets) to dissolve after an excess of water had been added. Moisture treatment of the ODTs with average PVP concentrations ( 50-60 wt\%) produced "soft rubber-like" slightly sticky tablets with disintegration time within 20-30 seconds. Similar results were obtained for all three amino acids tested.

\section{Mechanical and texture properties of the freeze-dried products}

Mechanical properties of the intact freeze-dried cakes were analyzed directly in glass vials by Texture Analysis using the procedure and settings developed in [21]. Fig. 5 ((a) and (b)) shows the representative TA "force vs. displacement" curves recorded for 3-excipient ODTs comprising mannitol, PVP30K and Gly or Ser. As is seen, the shapes of TA profiles vary significantly for different compositions. Formulations containing low PVP concentrations have relatively monotonous profiles characterised by an initial increase in the penetration force (and, consequently, stress) with strain (or time), since the deeper a probe moves, the higher force is required to penetrate / compress the material. At a certain time, a transition from a nonlinear elastic (initial part) behaviour to irreversible plastic deformation occurs and the increase in penetration force discontinues [34, 35].

Upon rising the PVP content, relatively smooth and monotonous TA curves become non-monotonous with a few fractures. FD formulations with high PVP30K content ( $>40 \%$ ) show remarkably different type of the TA profiles - jagged (or 'multi-peak') curves. This type of compressive force vs. displacement dependencies is typical for brittle/fragile products [36,37]. When a probe penetrating a FD cake causes a critical level of stress, the pore walls break and a brittle cake deforms (crack formation). The fracture leads to a rapid 
significant reduction in force/stress. It has been shown previously, that the degree of jaggedness can serve as a measure of brittleness, or fracturability [36, 37]. In its turn, fracturability correlates with cake cracking [38]. Previously [21] we have shown that the two-component FD cakes (mannitol-PVP30K and alaninePVP30K) show similar dependence of the TA profiles on the PVP content in cakes.

Pure mannitol FD cakes are very soft and easily penetrable by a cylindrical probe; the maximum stress value for mannitol cakes was below $\sim 3 \mathrm{~g} / \mathrm{mm}^{2}$ [21]. Two- and three-excipient FD formulations containing PVP and an amino acid form much harder cakes; the maximum stress values increase when the polymer content increases (Fig. 5 (c)). As expected, tablets containing 5\% solid are significantly less strong than those containing $10 \%$ solid for all amino acid tested.

The stress dependences on the PVP content are non-linear and the highest growth in the tablet hardness occurs in the 30-50 wt\% interval of the PVP concentrations (Fig. 5 (c)). Increase in the PVP concentrations above 60 wt\% does not show significant contribution into the tablet hardness (Fig. 5 (c)). However, it should be noted that the maximum stress values not always correctly describe the hardness of the cakes as a sharp drop(s) in the penetration force due to cake cracking can considerably bias the maximum values. As is seen from the TA curves (Fig. 5 ( $a$ and b)), high PVP concentrations strongly increase fracturability of the tablets producing jagged "force vs. displacement" profiles. A number of cracks can lead to underestimated "apparent" values of the stress in the case of brittle products.

Fig. 5 (c) also reveals the difference in the hardness of the tablets formed using different amino acids. Tablets containing Gly were slightly weaker than those containing Ala or Ser. Increase in the mannitol content in mannitol-PVP30K-Ala(Gly) formulations (from 20 wt\% presented in Fig. 5 to 40 wt\%) did not change the general character of the stress dependences on the PVP content; however, the stress values were $10-15 \mathrm{~g} / \mathrm{mm}^{2}$ lower (not illustrated). As mannitol itself forms very week FD cakes, it is likely that increase in its content in a tablet (and, consequently, decrease in the amino acid content) results in reduction of the tablet hardness.

In our previous work [21] we examined the applicability of several parameters to quantitatively describe the jaggedness of TA curves and how these parameters change when going from nearly monotonous to jagged curves:

- Number of positive (or negative) force peaks exceeded a specific threshold force value (which can be chosen to exclude the "noise" peaks) increases drastically for jagged curves;

- Linear distance (the length of a line which joins all experimental points measured) for jagged curves tends to be much longer when compared to a smooth line;

- Average gradient of all positive slopes in a selected region increases as a jagged curve has many steep slopes;

- Average drop off value (the drop-in force between 2 consecutive peaks averaged over a selected region) increases;

- Dispersion value (the sum of the absolute changes in force between each consecutive data point in the selected region) also increases as force changes significantly in a narrow interval [21].

Fig. 5 (d-f) shows the dependencies of the numbers of positive peaks, average gradient and drop off values on the PVP content for the 3-excipient ODTs containing mannitol (20 wt\% fixed), PVP30K and an amino acid 
(Ala, Gly or Ser). As expected, the number of peaks rises remarkably from less than 10 for low-PVP mainly smooth curves to more than 60-70 for jagged high-PVP curves (Fig. 5 (d)). In the current work $50 \mathrm{~g}$ threshold force was used in the calculations hence very small "noise" peaks were excluded from the quantitative analysis. Lowering the threshold force resulted in a proportional increase in the numbers of peaks for all samples not changing the general character of the dependencies given in Fig. 5.

An increase in the PVP content in the tablets (and transition from monotonous to jagged TA curves (Fig. 5 (a and b)) also results in significant increase in the values of average gradient, drop off (Fig. 5 (e-f)) and linear distance (not illustrated). As it was observed in the case of the number of peaks, the increase in these values occurs in the narrow PVP concentration intervals for all tree amino acid studied. Samples containing $5 \%$ solids showed less increase in the linear distance, average gradient and drop off values with PVP concentration than samples containing $10 \%$ solids.

It should be noted that low rate of probe penetration, suggested in our previous work [21], permits studying the hardness as well as the texture and the vertical heterogeneity of FD cakes. However, some "imperfections" on the top of a cake (for instance, "skin" formation or "volcanos" [31 and references therein]) can slightly bias the initial part of a TA profile ( $1 \mathrm{sec})$ as they prevent from the full flat contact between a probe and the top surface. Such imperfections, even if clinically irrelevant, should be avoided when developing ODTs, as the tablet appearance is considered amongst the critical quality attributes for the ODTs.

To assess the effect of a small increase in moisture on the texture properties, 3-excipient tablets containing mannitol (fixed $40 \mathrm{wt} \%$ ), PVP and either Ala or Gly were prepared, moisturised and examined by TA. As is seen in Fig. 6 (a), moisture treatment changes the shape of the TA profiles making jaggedness significantly more pronounced even at low and medium PVP concentrations. As a result, the number of peaks (Fig. 6 (b)), linear distances, average gradients (Fig. 6 (c)) and drops off (Fig. 6 (d)) increase sharply for the moist tablets. The increase occurs in the narrow intervals of the PVP concentrations for both amino acids. Moist tablets also become stronger than the dry tablets as they require higher force to penetrate a tablet (Fig. 6 (e)). It is of interest to mention that after moisture treatment Gly-containing tablets with low PVP content (10-30 wt\%) possess some degree of elasticity (characterised by increase in the values of stress relaxation (see Materials and Methods) from about 1 to 1.2-1.3 (not illustrated)).

\section{Thermal behavior of the freeze-dried formulations}

Fig. 7 (a) (curves 5-7) shows the DSC thermograms of 3-excipient FD formulations comprising mannitol, PVP30K and serine with different serine-to-PVP ratios. For a comparison, the melting curves of freeze-dried pure mannitol, PVP30K and serine are shown (Fig. 7 (a), curves 1-3).

As is seen, the DSC thermogram of FD serine has one asymmetric endothermic melting peak in the region of 220-250 ㄷ confirming that after lyophilisation pure serine is in crystalline form (Fig. 7 (a), curve 3). The apparent melting enthalpy calculated from the DSC thermograms (Fig. 7 (a), curves 4-7) decreases as the amount of serine in 3-excipient formulations decreases (Fig. 7 (b), curve 1). However, the corrected values of the $\Delta \mathrm{H}_{\text {melt }}$ (Fig. 7 (b), curve 2), where the actual Ser concentrations in the formulations were taking into account, are shown to be almost independent on the serine contents in the 3-excipient FD cakes. This 
evidences that serine remains in the crystalline state in formulations with different PVP30K concentrations. Similar results were obtained for the 3-excipient formulations containing different amino acids (Ala or Gly).

A strong endothermic peak around $150-160$ ㅇ (Fig. 7 (a), curve 2) evidences that pure FD mannitol is in crystalline form after lyophilisation. In the freeze-dried 3-excipient formulations this peak shifts toward lower temperatures, becomes smaller and eventually disappears when PVP30K content increases above $60 \mathrm{wt} \%$ (Fig. 7 (a), curves 4-7, and (b), curve 3). Similar tendency has been shown for 3-excipient formulation containing $40 \mathrm{wt} \%$ of mannitol, Gly and PVP30K (not illustrated). Melting peak disappearance indicates a partial mannitol conversion into an amorphous form in the PVP-rich formulations. It should be noted, that, unlike serine, mannitol concentration in the 3-excipient formulations was kept constant (Table 1). To verify the PVP30 effect on the state of mannitol, two-excipient formulations containing different concentrations of mannitol and PVP30K were freeze-dried and analysed by DSC. The results obtained show that the melting peak of mannitol decreases significantly when PVP content rises above 60 wt\% (Fig. 8) thus supporting the conclusion that mannitol in the PVP30K-rich samples is in amorphous state.

\section{Discussion}

It was shown by us previously [39], that mannitol in a combination with amino acids (in particular, Larginine) forms very elegant freeze-dried cakes. The present results also show that addition of small amounts of mannitol ( $20 \mathrm{wt} \%$ ) helps improving the external appearance and producing visually elegant lyophilized ODTs comprising PVP and amino acids. Despite PVP-AA tablets were un-collapsed and relatively well-shaped, a number of cracks visible near the walls (Fig. 1), especially in the case of Gly-PVP tablets, can potentially make the final product less attractive for the patients (as "non-ideal" cake appearance is often an apparent indicator of a poor formulation or processing even if it has no impact on product quality [31 and references therein]). Partial substitution of mannitol with an amino acid can, therefore, reduce the polyol content in the tablets and preserve the quality of the final product.

The type of an amino acid can also contribute towards the quality of the freeze-dried tablets. Our results show that Ala-containing cakes are, in general, better adhered to the vials and Gly-containing cakes had worse adherence. However, only the ratio between mannitol and PVP concentrations had a significant impact on the visual quality of the tablets.

Up to the date there are several experimental methods to assess the disintegration time of the orally disintegrating tablets since the Pharmacopoeia disintegration test used for regular tablets (which requires a large volume of water ( $>500 \mathrm{~mL}$ ) and rapid and vigorous agitation) does not accurately represent the conditions of the oral cavity [40]. In the present work we used $1 \mathrm{~mL}$ of colored water as the average rate of saliva secretion is $\sim 0.2-0.4 \mathrm{~mL} / \mathrm{min}$ when resting, although it could be increased up to $2 \mathrm{~mL}$ upon stimulation [40; 41]. According to the Guidance for Industry: Orally Disintegrating Tablets published by the US Food and Drug Administration, ODTs should have an in vitro disintegration time of approximately $30 \mathrm{sec}$. or less (using United States Pharmacopeia disintegration test or equivalent) [42, 43, 27]. The European Pharmacopeia has used the term orodispersible tablets for uncoated tablets that disperse/disintegrate within $3 \mathrm{~min}$ when placed in the mouth before swallowing [27]. It is assumed that tablets can disintegrate into smaller granules or melts into an easy swallowing gel-like structure. 
The results obtained in the present work show that all the 3-excipient tablets studied had the disintegration times of less than $30 \mathrm{~s}$ thus complying with FDA recommendations for ODTs. Mannitol replacement with amino acids does not compromise the disintegration of the lyophilized ODTs.

The presence of mannitol also reduces the moisture uptake by the freeze-dried formulations as mannitol has very good moisture-resistant properties even complexed with highly hygroscopic materials (for instance, L-arginine hydrochloride [39]). The moisture uptake by the ODTs studied was shown to be dependent mostly on the PVP content and increased with the PVP concentration in the formulations (Fig. 4). The results on the moisture-induced shrinkage of the freeze-dried cakes reveal that high PVP content in a formulation also leaded to a higher percentage of cake shrinkage which is proportional to the decrease in the cake diameter (Fig. 3). Two-component FD cakes without mannitol showed increased level of shrinkage, thus it can be concluded that the presence of low hygroscopic mannitol (even in a low concentration) reduces the tablet sensitivity to humidity. This moisture protection is especially important since many highly watersoluble excipients are used in ODT formulations to enhance fast-dissolving properties as well as to create good mouth feel [20].

Moisture treatment of the ODTs in vitro at high relative humidity also imitates the limited saliva conditions in the mouth thus providing valuable insight into the condition of the partially moisturized tablets. ODTs with large PVP content (PVP-rich) become significantly harder adopting a "hard rubber-like" or "toffee-like" consistence after being slightly wetted. In addition, PVP-rich tablets convert into a sticky (and for some formulations, very sticky) products. The latter might affect patient compliance. Analysis of the results also shows that the consistence of the moist FD cakes is defined by the PVP content (or PVP-to-amino acid ratio) rather than by the type of an amino acid. Similar result has been shown for the total moisture uptake by the 3-excipient formulations (Fig. 4).

In addition to producing less elegant FD cakes and attracting more moisture, high concentrations of PVP in the 3-excipient formulations containing different amino acids also prevent mannitol from transition into a crystalline state during freeze-drying (Fig. 7) indicating a suppressive effect on mannitol crystallization. Probably, a small amount of mannitol in the formulation is solubilised by PVP preventing from crystallization. Incomplete mannitol crystallization may compromise the stabilizing effect of the crystalline mannitol on the lyophilized cakes and can result in product collapse [44]. The presence of an amino acid had no impact on the PVP-mannitol interaction as PVP also suppressed mannitol crystallization in the 2-component PVPmannitol systems (Fig. 8). An amino acid in the 3-excipient formulation remained in the crystalline state (Fig. 7), hence there is no strong interaction between an amino acid and mannitol or PVP.

As expected, the hardness of the freeze-dried tablets increases with the PVP content (Fig. 5). Comparison of the stress values for PVP-alanine-mannitol and PVP-alanine (briefly studied in [21]) FD cakes shows that the 3-excipient cakes are less strong due to the presence of very week mannitol. Our results also show that the hardness depends on the PVP content non-linearly - tablets with PVP above 30-40 wt\% are significantly stronger than those with less PVP; however, further increase in the polymer content makes less contribution to the tablet hardness (Fig. 5).

An increase in the PVP content in tablets affects not only the tablet hardness. In the $30-40 \mathrm{wt} \%$ PVP range another significant change in the texture profiles of the tablets occurs - a shift from the relatively smooth 
monotonous "stress-strain" TA curves to the jagged multi-peaks curves. As a result, a dramatic increase in the number of peaks, average gradient and drop off values was observed (Fig. 5 (d-e)). The transition between two types of the TA profiles ("relatively smooth" to "jagged"), and, hence, the increase in the peak number, average gradient and drop off values, occurs in a narrow interval of the PVP concentrations regardless of the type of an amino acid in the formulations both in the case of dry and moisturized tablets (Fig. 5 and 6). This means that the texture properties of the tablets depend non-linearly on the excipient composition. It seems likely that the polymer concentration heavily defines the texture of the lyophilized products. In our recent work [21] we have studied the texture properties of 2-excipient formulations PVP3OK - mannitol and PVP3OK - alanine. Two-excipient formulations also showed a shift from a smooth to a jagged type of the TA profiles in a narrow interval of PVP concentrations. This finding supports the suggestion that the amount of PVP in the tablets is mainly responsible for the tablet texture properties.

The results presented in Figs. 5 and 6 are in good agreement with our data on the moisture-induced cake shrinkage (Fig. 3) where a non-linear relationship between shrinkage and the PVP content was shown. The non-linear effect of the PVP content on different tablet properties should be taken into account when designing ODT formulations comprising PVP and mannitol. This finding can be especially important for the QbD approach [45] as the existence of a narrow "yes-no" PVP interval (where a small variation in the polymer content results in different texture properties of FD cakes) can compromise robustness of the manufacturing process.

Overall, the results obtained show that mannitol can be partially replaced with amino acids in the ODT formulations. The replacement improves tablet strength and has only a limited impact on the tablet texture properties and disintegration. Ala, Gly and Ser formed similar yet not identical 3-excipient (mannitol-PVPAA) FD cakes with minor differences in the moisture uptake, shrinkage and the texture properties. The results show that alanine made FD cakes harder and slightly more resistant to moisture-induced shrinkage than glycine. At the same time Ala-containing tablets showed more jagged TA profiles (possessed higher values of average gradient, linear distance, etc.) meaning that alanine increases fracturability of the FD tablets. However, the exact effect of each amino acid on the FD tablet structure and properties requires further investigations. Whilst the dry products containing different amino acids might expose only a minor variance between them, the moisture treatment of the FD cakes revealed more sizeable differences between AA-containing cakes, especially at low PVP concentrations when amino acid content is higher (Fig. 6). This approach can be used in future works to highlight and make more conspicuous small variances between formulations containing different amino acids.

It is of interest to note that, despite having rather different TA profiles (Figs. 5 and 6), both low- and highPVP tablets possess the look-alike internal closed-cell hexagonal structures (Fig. 2) with comparable values for cell dimensions and cell wall thicknesses. Similar result was obtained in our previous work [21] when analysing the internal structures and the TA profiles of different mono-excipient freeze-dried cakes. It seems that the strength of the cakes is determined not only by the size of the pores and walls but also by the composition of the walls. These results show that the texture analysis provides independent valuable information on the texture of the freeze-dried samples. The present study also confirms that the TA method is sensitive to the variations in the physical properties of the FD cakes that occur due to cake preparation or/and storage conditions (e.g. excessive moisture). 
The texture analysis approach developed in our previous work [21] and applied in the current study is a very simple yet robust method to assess the texture properties of the freeze-dried cakes without the necessity to damage or alter a cake in any way before the experiment. The procedure is sensitive to the product composition and allows quantitative assessment of the strength, fracturability and elastic properties of the FD cakes or tablets. Assessing the mechanical properties of the freeze-dried cakes is especially important when developing orally disintegrating tablets as they have to possess a short disintegration time and adequate mechanical properties. It is not an all-purpose requirement for a freeze-dried cake to be sufficiently strong; and some lyophilized injectable pharmaceutics can form "imperfect" cakes without any impact on the clinical properties [31]. Moreover, an innovative continuous freeze-drying technology for unit doses suggested recently implies a rapid rotation of the vials along their longitudinal axis so the resulting lyophilized product does not form a cake at all but is spread over the entire vial surface [46]. However, for some products, and, in particular, for the orally disintegrating tablets, the mechanical strength of a tablet is amongst the critical quality attributes. In this case the TA measurements directly in freeze-drying glass vials can be routinely used in the formulation development. A variety of the TA probes on the market allows choosing an optimal probe for a particular application. At the same time developing the ODT formulations is not the only area where the suggested TA approach can be applied. It can also give an important information on the FD cake quality and freeze-dried cycle reliability on the formulation development stage. According to authors of [47], around 50\% of the FDA and EMA approved biopharmaceutical products (over 300 to date) are freeze-dried making this technique one of the preferred ways of stabilizing biopharmaceutical drug products unstable in aqueous solution.

\section{Conclusion}

The results show that appearance, the mechanical properties, disintegration and shrinkage of the FD orally disintegrating tablets formed by PVP30K, mannitol and an amino acid depend significantly on the excipients composition with PVP playing the leading role. Partial mannitol replacement with an amino acid has a limited impact on the tablet properties. The presence of an amino acid also has no impact on the PVPmannitol interaction. Moisture treatment can make small variances between formulations containing different amino acids more conspicuous.

ODTs with high PVP content are slightly less elegant, absorb more moisture and shrink faster and to a higher extend than the tablets with a moderate PVP content. Moisturised high-PVP tablets become hard, sticky and possess longer disintegration times that can be undesirable for users. In addition to producing less elegant FD cakes and attracting more moisture, high concentrations of PVP show a suppressive effect on mannitol crystallization. At the same time the high PVP concentrations do not contribute more into the tablet hardness and texture properties than the average PVP concentrations.

Depending on the PVP content, freeze-dried tablets studied reveal two types of the texture profiles smooth or jagged multi-peaks TA curves. The transition between the two types of textures occurs in a narrow interval of PVP concentrations regardless of the type of amino acid in a formulation both in the case of dry and partly moisturized tablets. That means that the texture depends non-linearly on the PVP content. Comparison of the mechanical and texture properties of PVP-mannitol and PVP-mannitol-AA tablets shows that the polymer is mainly responsible for the tablet texture properties. The non-linear effect of PVP on 
different tablet properties should be taken into account when designing ODT formulations as it can compromise the robustness of the manufacturing process.

The texture analysis approach applied in the current study is a very simple yet robust method to assess the texture properties of the freeze-dried cakes without the necessity to damage or alter a cake in any way before the experiment. The procedure is sensitive to the product composition and allows quantitative assessment of the strength, susceptibility to breakage, fracturability and elastic properties of the FD cakes or tablets.

\section{Acknowledgments}

The authors would like to thank Dr. Rachel Armitage (De Montfort University) for help with collecting SEM images. 
Table 1. Compositions of ODT formulations studied (Mannitol is referred to as Man, AA - amino acid, PVP Polyvinylpyrrolidone 30K).

\begin{tabular}{|c|c|c|c|c|c|c|}
\hline $\begin{array}{l}\text { Formulation } \\
\text { composition }\end{array}$ & $\begin{array}{l}\text { Number of } \\
\text { excipients }\end{array}$ & $\begin{array}{l}\text { Amino } \\
\text { acids } \\
\text { studied }\end{array}$ & $\begin{array}{c}\text { Total solid } \\
\text { material, } \\
\text { wt \% }\end{array}$ & $\begin{array}{c}\text { Mannitol, } \\
\%\end{array}$ & $\begin{array}{l}\text { Amino } \\
\text { acid, \% }\end{array}$ & PVP, \% \\
\hline Man + AA + PVP & 3 & $\begin{array}{l}\text { Ala } \\
\text { Gly } \\
\text { Ser }\end{array}$ & $5 \%$ & $20 \%$ fixed & $\begin{array}{l}\text { from } 0 \text { to } \\
80 \%\end{array}$ & $\begin{array}{l}\text { from } 80 \text { to } \\
0 \%\end{array}$ \\
\hline Man + AA + PVP & 3 & $\begin{array}{l}\text { Ala } \\
\text { Gly } \\
\text { Ser }\end{array}$ & $10 \%$ & $20 \%$ fixed & $\begin{array}{l}\text { from } 0 \text { to } \\
80 \%\end{array}$ & $\begin{array}{l}\text { from } 80 \text { to } \\
0 \%\end{array}$ \\
\hline Man + AA + PVP & 3 & $\begin{array}{l}\text { Ala } \\
\text { Gly }\end{array}$ & $10 \%$ & $40 \%$ fixed & $\begin{array}{l}\text { from } 0 \text { to } \\
60 \%\end{array}$ & $\begin{array}{l}\text { from } 60 \text { to } \\
0 \%\end{array}$ \\
\hline Man + PVP & 2 & none & $10 \%$ & $\begin{array}{l}\text { from } 10 \text { to } \\
90 \%\end{array}$ & - & $\begin{array}{l}\text { from } 90 \text { to } \\
10 \%\end{array}$ \\
\hline$A A+P V P$ & 2 & $\begin{array}{l}\text { Ala } \\
\text { Gly }\end{array}$ & $10 \%$ & - & $\begin{array}{l}\text { from } 10 \text { to } \\
90 \%\end{array}$ & $\begin{array}{l}\text { from } 90 \text { to } \\
10 \%\end{array}$ \\
\hline
\end{tabular}

\begin{tabular}{llll}
20 & 20 & 20 & 20 \\
70 & 50 & 30 & 10 \\
10 & 30 & 50 & 70 \\
\hline & & & \\
\hline
\end{tabular}
Mannitol, wt\%
Ala, wt\%
PVP30K, wt\%

Fig. 1. Representative images of lyophilized cakes comprising mannitol (20 wt\%), alanine (Ala) and PVP30K. Samples were freeze-dried and stoppered under vacuum conditions. All samples contain $10 \% \mathrm{w} / \mathrm{w}$ solid material. 


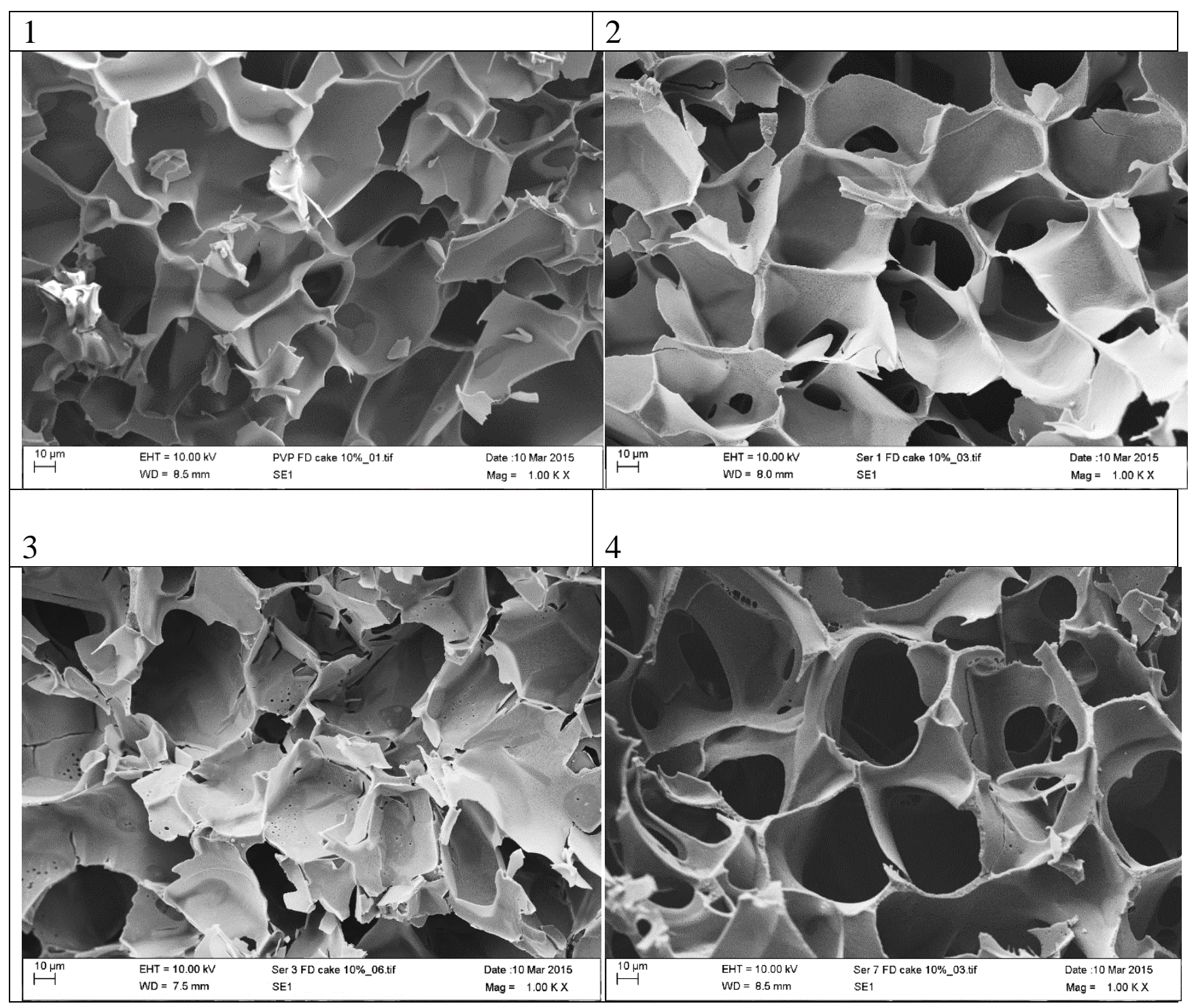

Fig. 2. SEM images (x1000 magnification) of lyophilized cakes comprising

(1) pure PVP30K;

(2) mannitol (20 wt\%) and serine (80 wt\%)

(3) mannitol (20 wt\%), PVP30K (20 wt\%) and serine (60 wt\%)

(4) mannitol (20 wt\%), PVP30K (60 wt\%) and serine (20 wt\%)

All samples were prepared from $10 \%$ solid in liquid solutions. 
a).

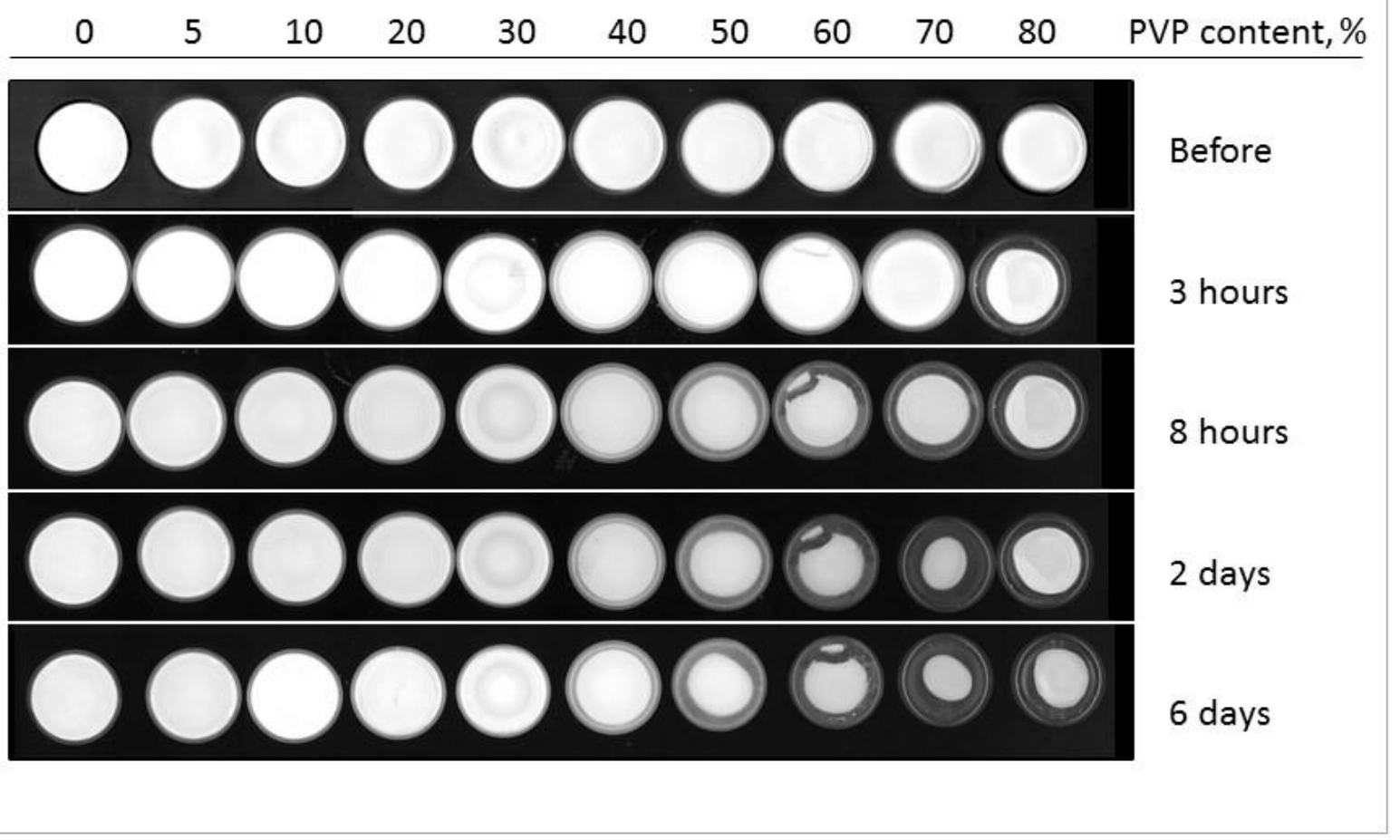

b).

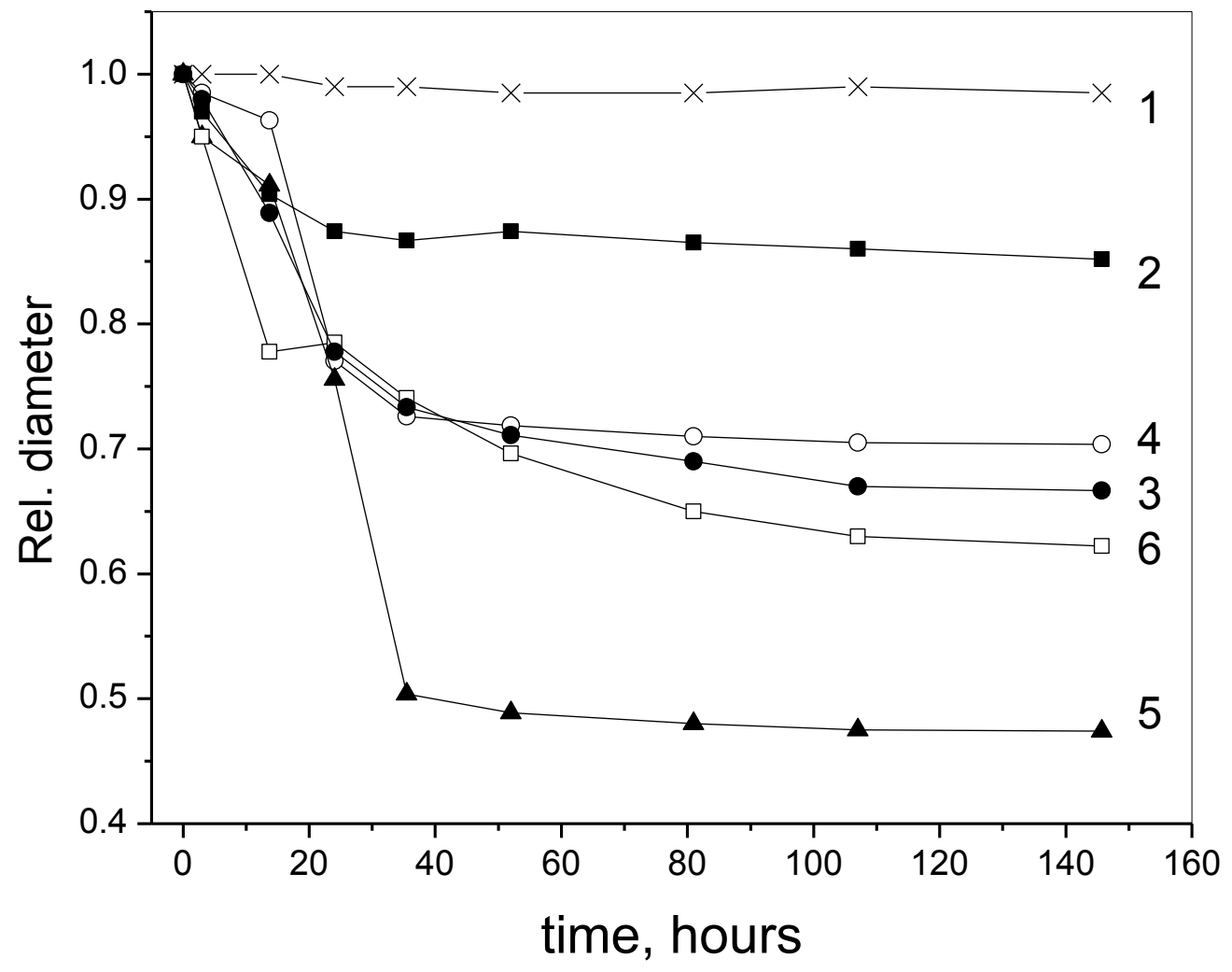


c).

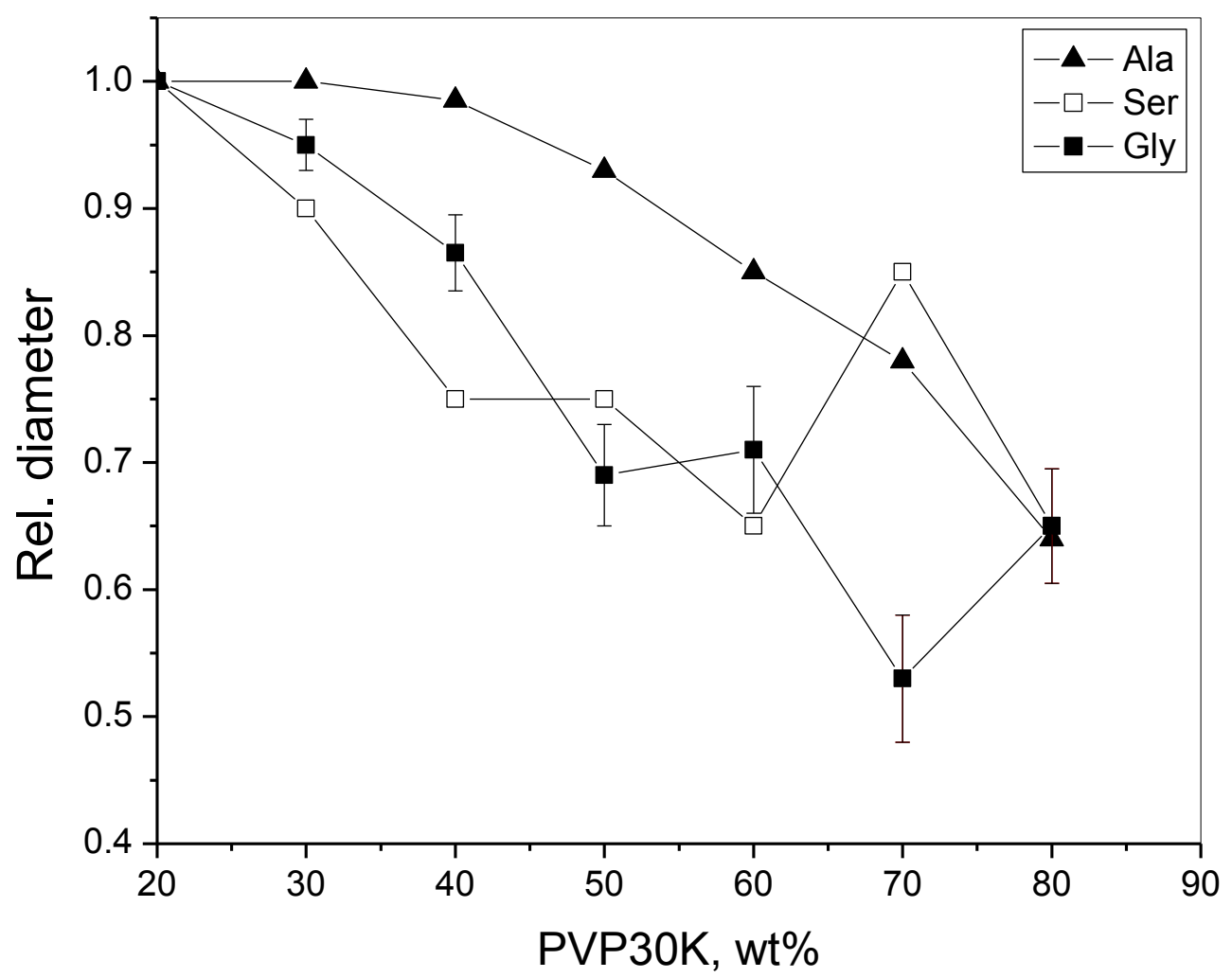

Fig. 3. Moisture-induced shrinkage of lyophilized cakes comprising mannitol, PVP3OK and an amino acid (Ala, Gly or Ser). Concentration of mannitol was fixed at $20 \mathrm{wt} \%$; concentrations of PVP3OK and an amino acid (AA) were varied (PVP30K $+A A=80 \mathrm{wt} \%$ ). Cakes were incubated at $75 \% \mathrm{RH}$ at room temperature.

a). Scans of glass vials (bottom view) containing FD products (Mannitol + PVP30K + Gly) taken before (time 0 ) and during moisture treatment (the incubation times are indicated).

b). Dependencies of the relative diameters of FD cakes on time of incubation at $75 \% \mathrm{RH}$ for formulations containing mannitol (20 wt\%), PVP30K and Gly. PVP30K concentrations, wt $\% ; 1-30 ; 2-40 ; 3-50 ; 4-60 ; 5$ - 70; 6 - 80. Relative diameter values were calculated as ratios between the cake diameters after and before the moisture treatment.

c). Dependencies of the relative diameters of FD cakes on the PVP content in formulation after $82 \mathrm{~h}$ of moisture treatment. 
Fig. 4.

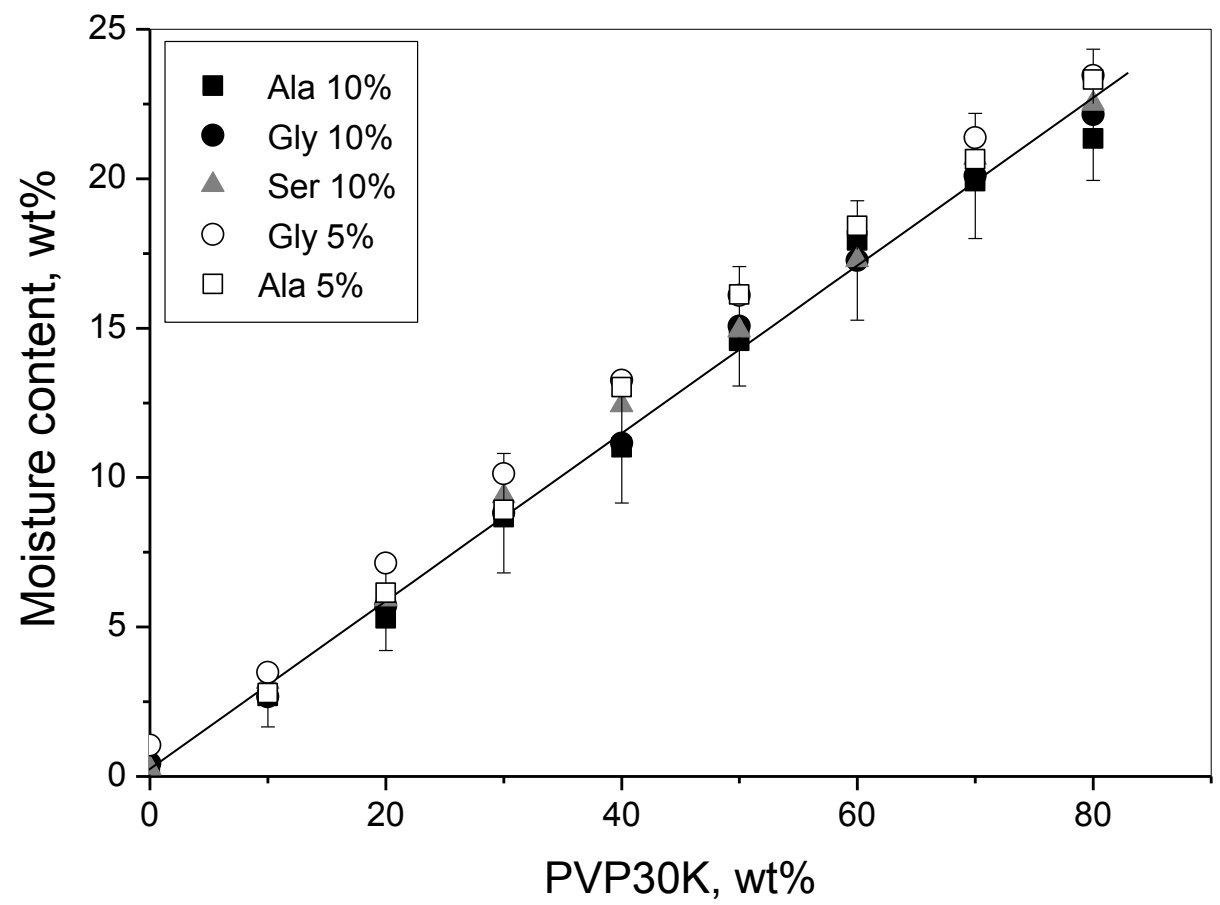

Fig. 4. Dependences of the total moisture uptake by lyophilized ODTs on the PVP3OK content in tablets after 6-days incubation at $75 \% \mathrm{RH}$ at room temperature. ODTs content: mannitol (20 wt\%), amino acid (Ala, Gly or Ser) and PVP30K; the total solid content was $5 \%$ or $10 \%$. Error bars and linear regression line are shown for Gly $10 \%$ samples only. 
Fig. 5.

a).

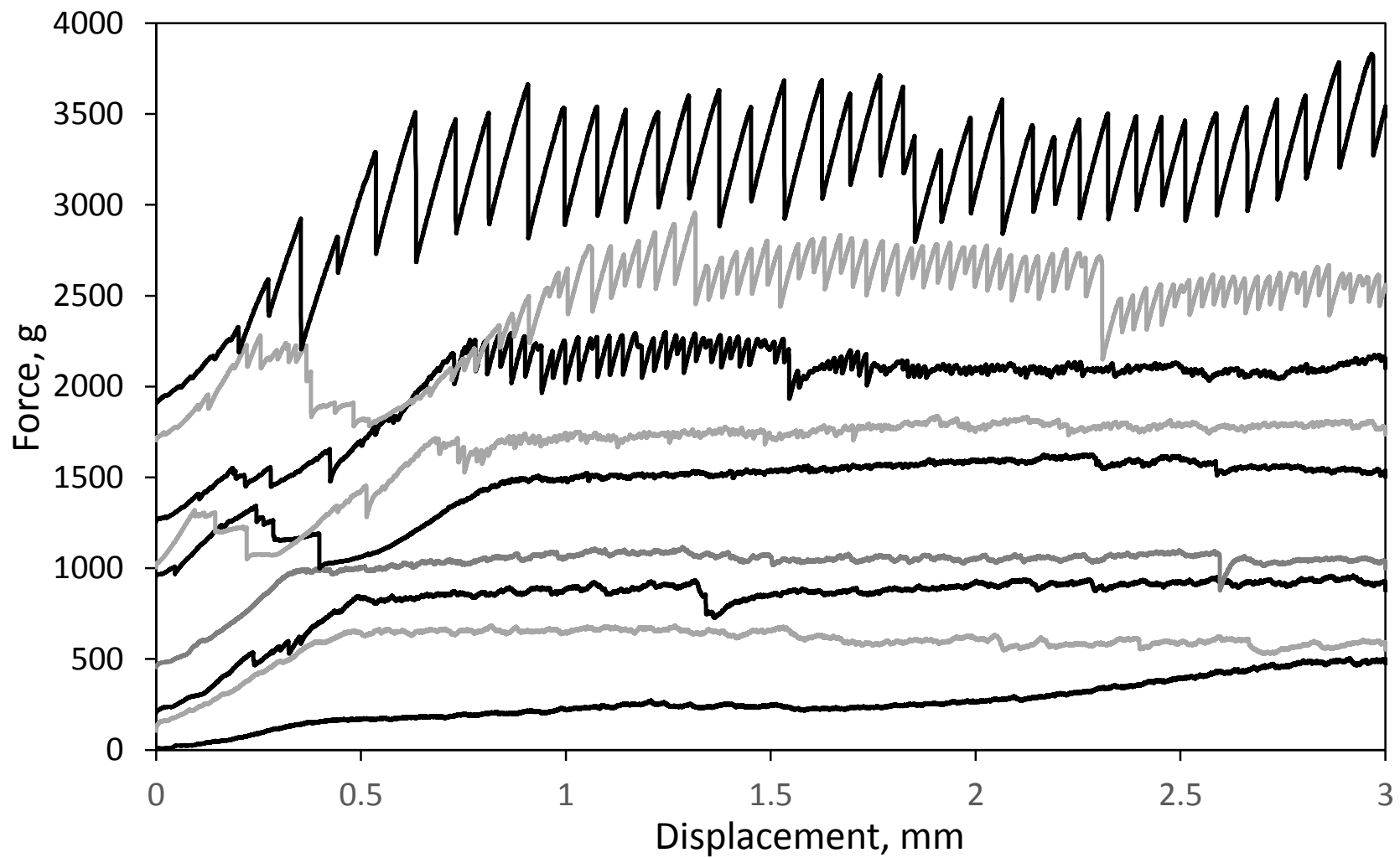

b).

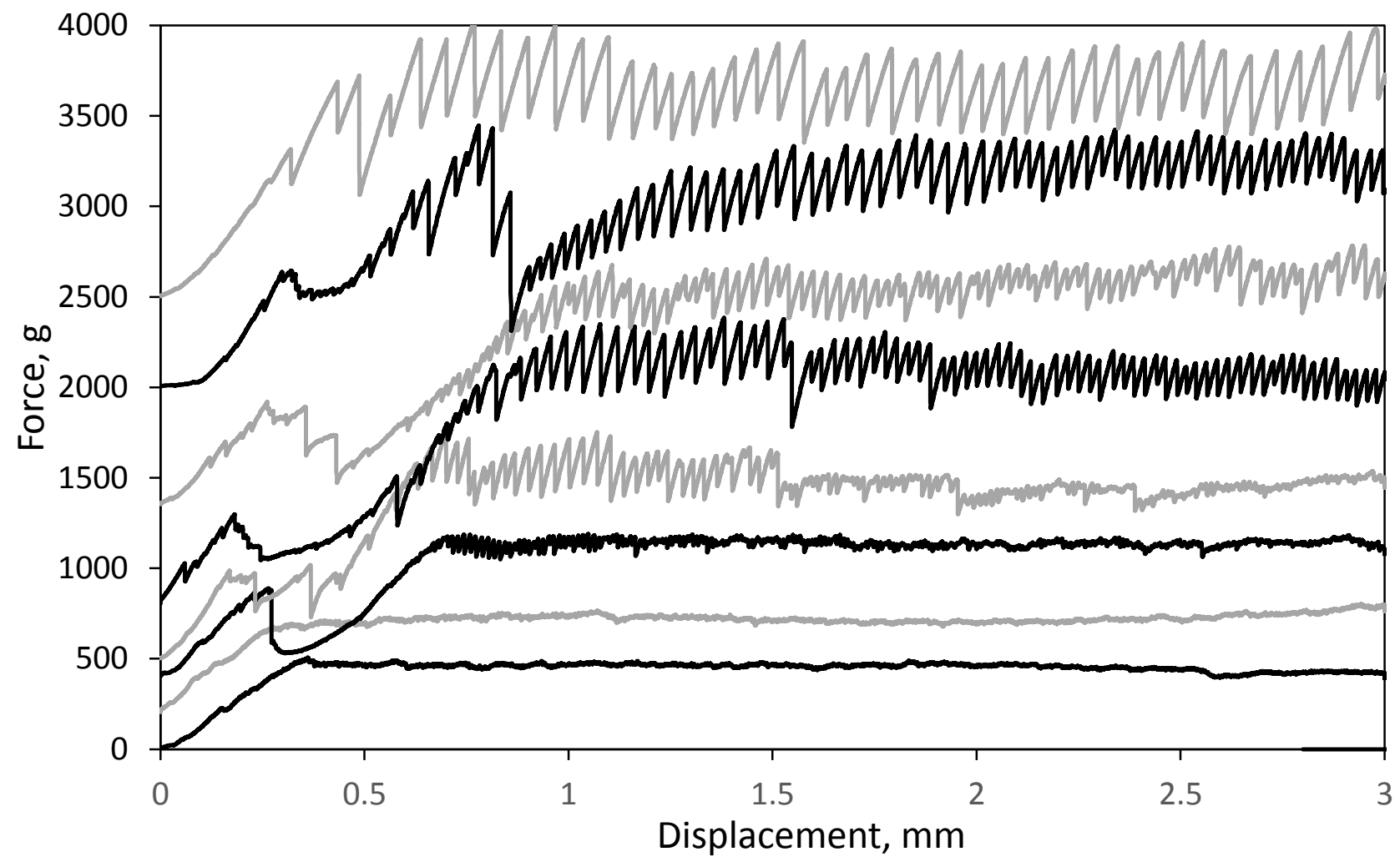


c-f).

c).

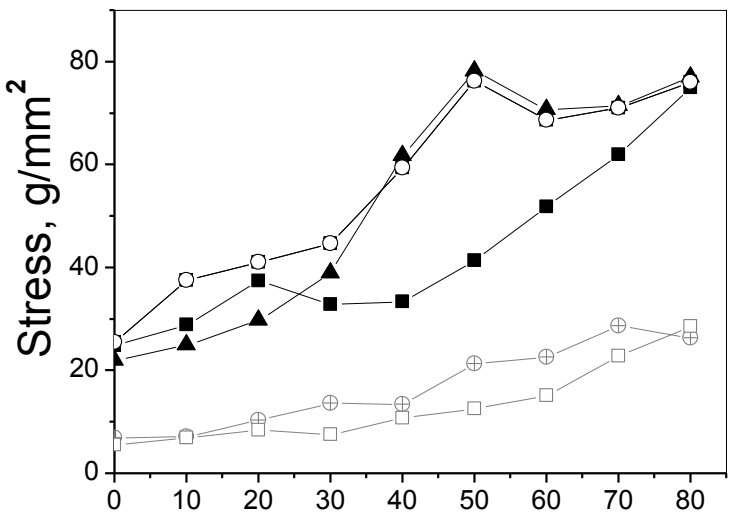

d).

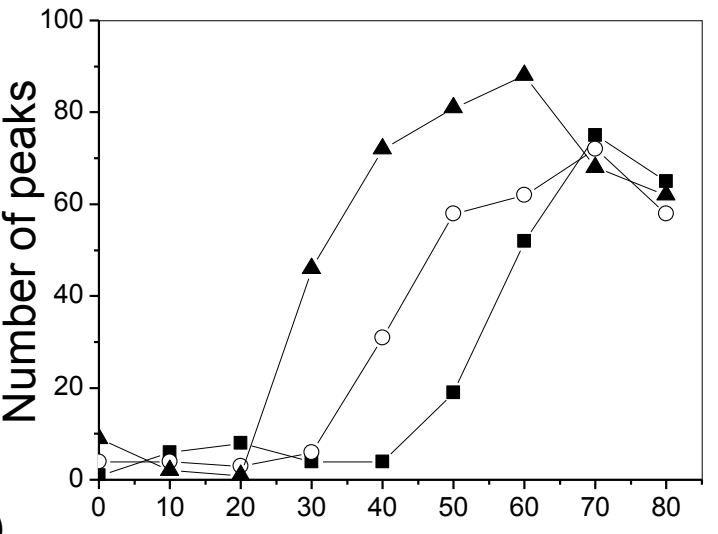

e).
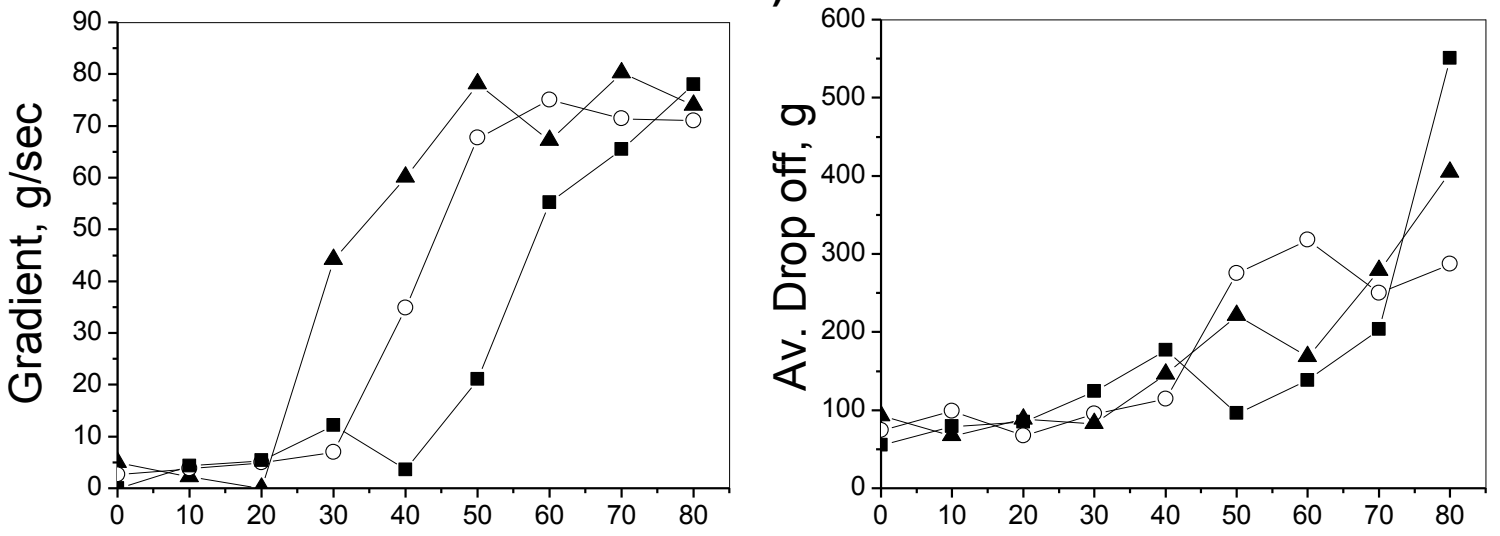

f).

\section{PVP concentration, wt \%}

Fig. 5. Mechanical and texture properties of lyophilized 3-excipient tablets comprising mannitol (fixed 20 wt\%), PVP30K and an amino acid (Ala, Gly, or Ser). The total solid content, if not specified, was $10 \%$.

(a) and (b): "Force vs. displacement" TA curves for formulations containing Gly (a) and Ser (b). PVP concentrations, wt\% (from bottom to top): (a) 0, 10, 20, 30, 40, 50, 60, 70, 80; (b) 10, 20, 30, 40, 50, 60, 70, 80. The Force scale $(\mathrm{Y})$ is related to the bottom curve only, other curves are arbitrary shifted up.

(c). Dependence of the maximum stress on the PVP content in formulations containing Gly $(1,5)$, Ser $(2)$ or Ala $(3,4)$. The total solid content was $10 \%(1-3)$ or $5 \%(4,5)$.

$(d-f)$. Dependencies of the number of positive peaks $(d)$, average gradient (e) and average drop off (f) on the PVP content in formulations containing Gly (1), Ser (2) or Ala (3). 
Fig. 6.

a).
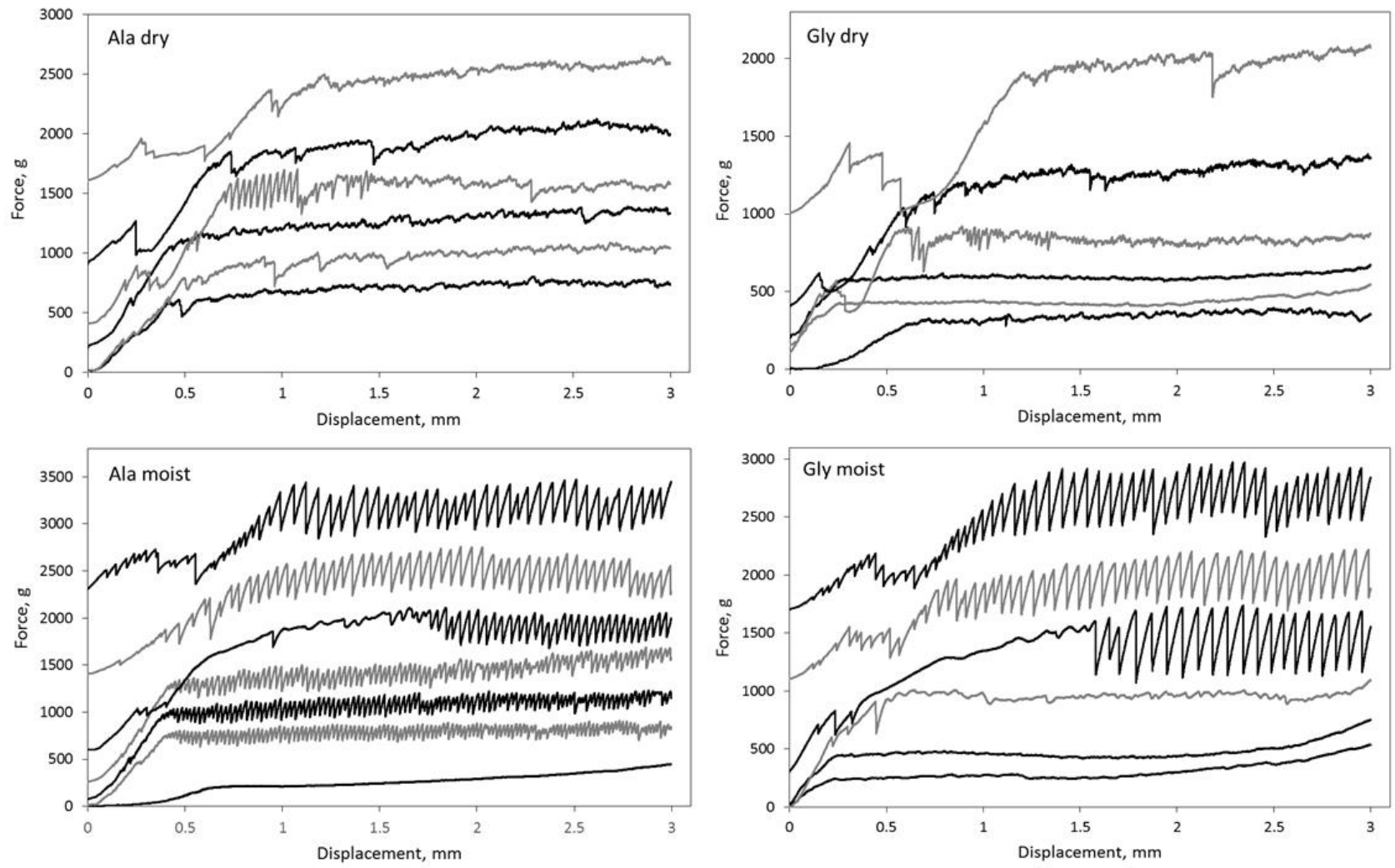
b).
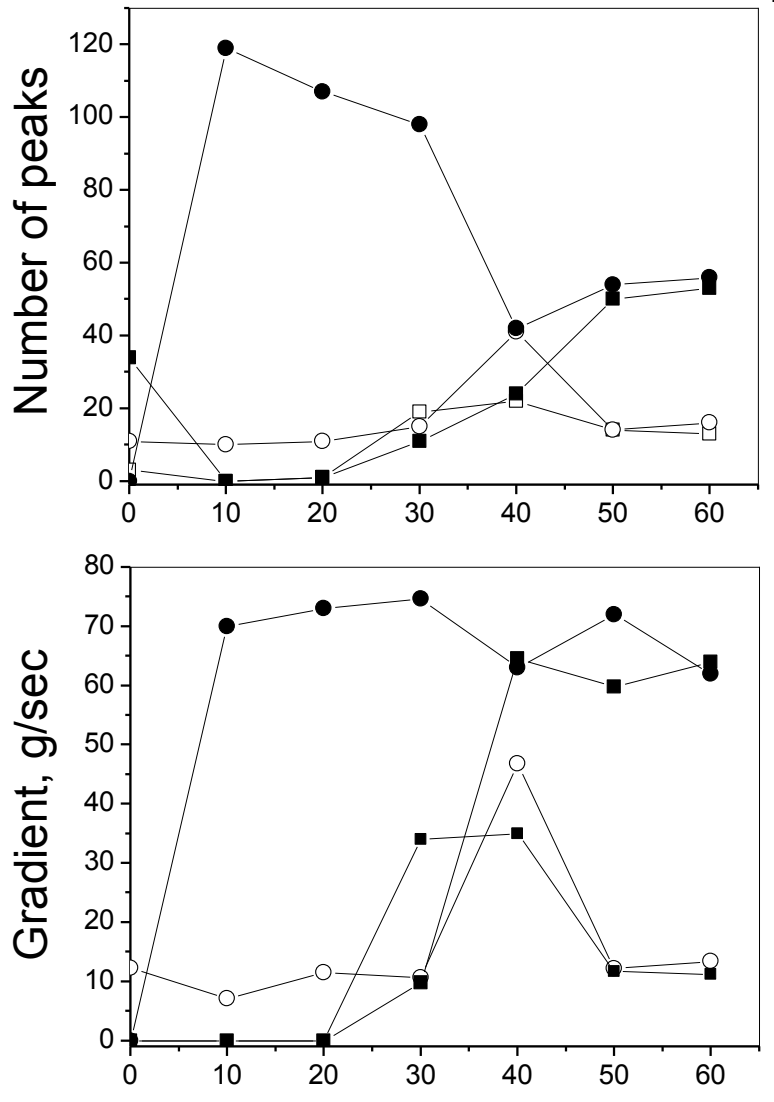

c).

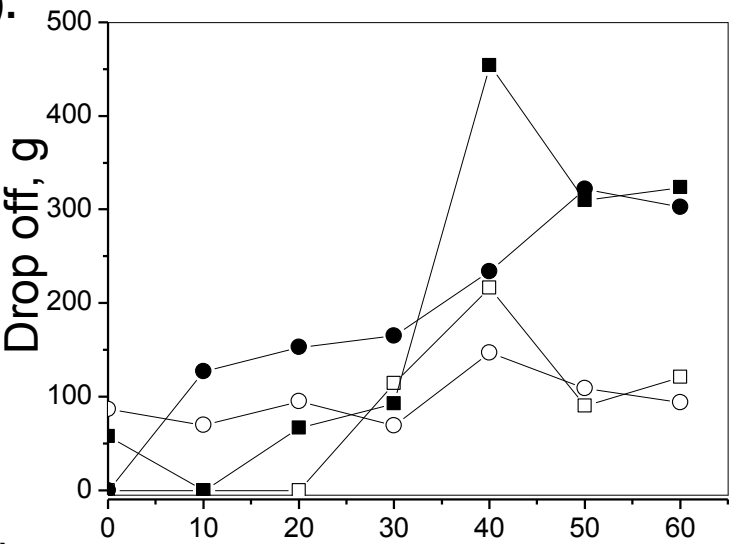

d).

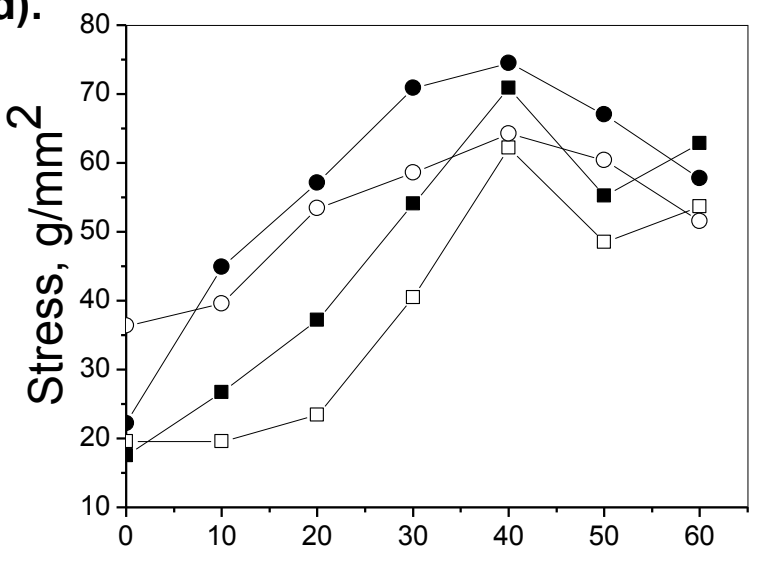

e).

\section{PVP concentration, wt\%}

Fig. 6. Effect of moisture treatment on the mechanical and texture properties of lyophilized 3-excipient ODTs comprising mannitol (fixed $40 \mathrm{wt} \%$ ), PVP30K and amino acid (Ala or Gly). Tablets were incubated at room temperature and $75 \% \mathrm{RH}$ during 1 hour prior to the TA measurements.

(a): "Force vs. displacement" TA curves for dry and moisturised tablets. PVP concentrations, wt\% (from bottom to top): Ala dry - 10, 20, 30, 40, 50, 60; Ala moist - 0, 10, 20, 30, 40, 50, 60; Gly dry - 0, 10, 20, 30, 50,60 ; Gly moist $-10,20,30,40,50,60$. The Force scale $(\mathrm{Y})$ is related to the bottom curve only, other curves are arbitrary shifted up.

$(b-e)$ : Dependences of the number of peaks (b), average drop off $(c)$, average gradient $(d)$ and maximum stress (e) on the PVP content in FD formulations. Values were calculated from the TA profiles using TA Exponent software with force threshold equal to $50 \mathrm{~g}$. 
Fig. 7.

(a)

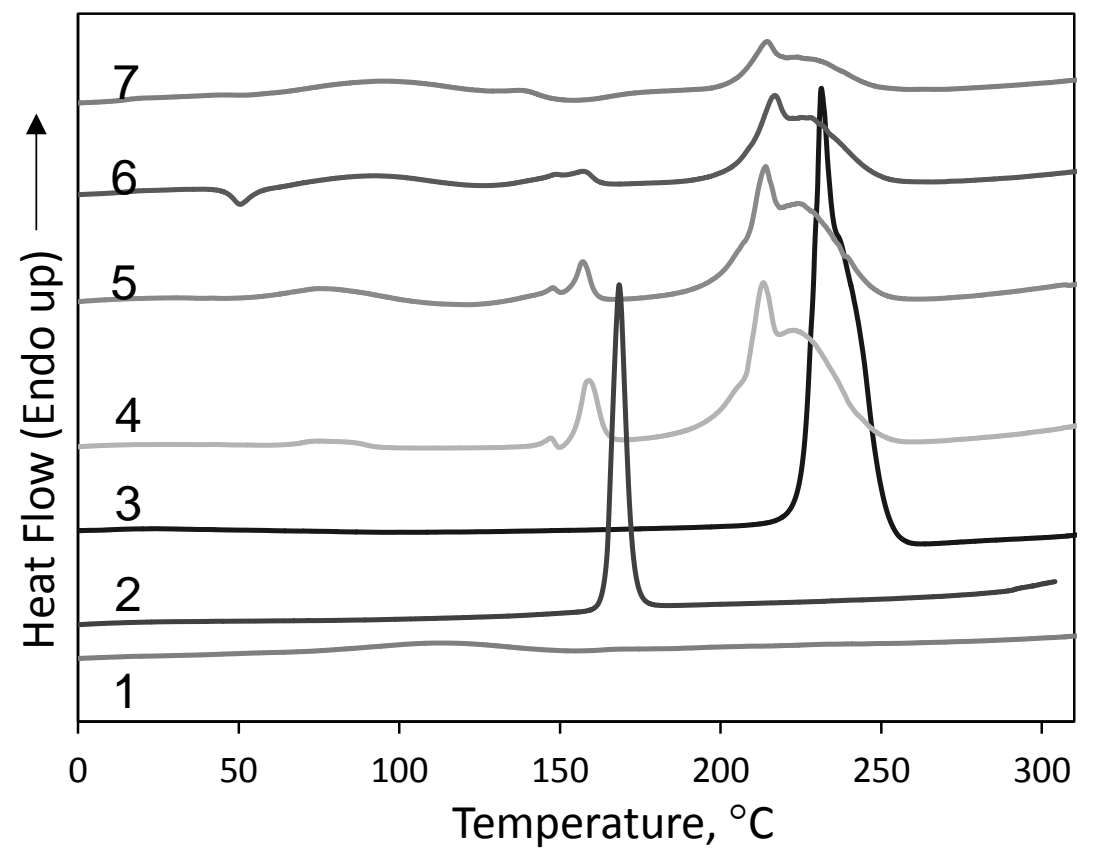

(b)

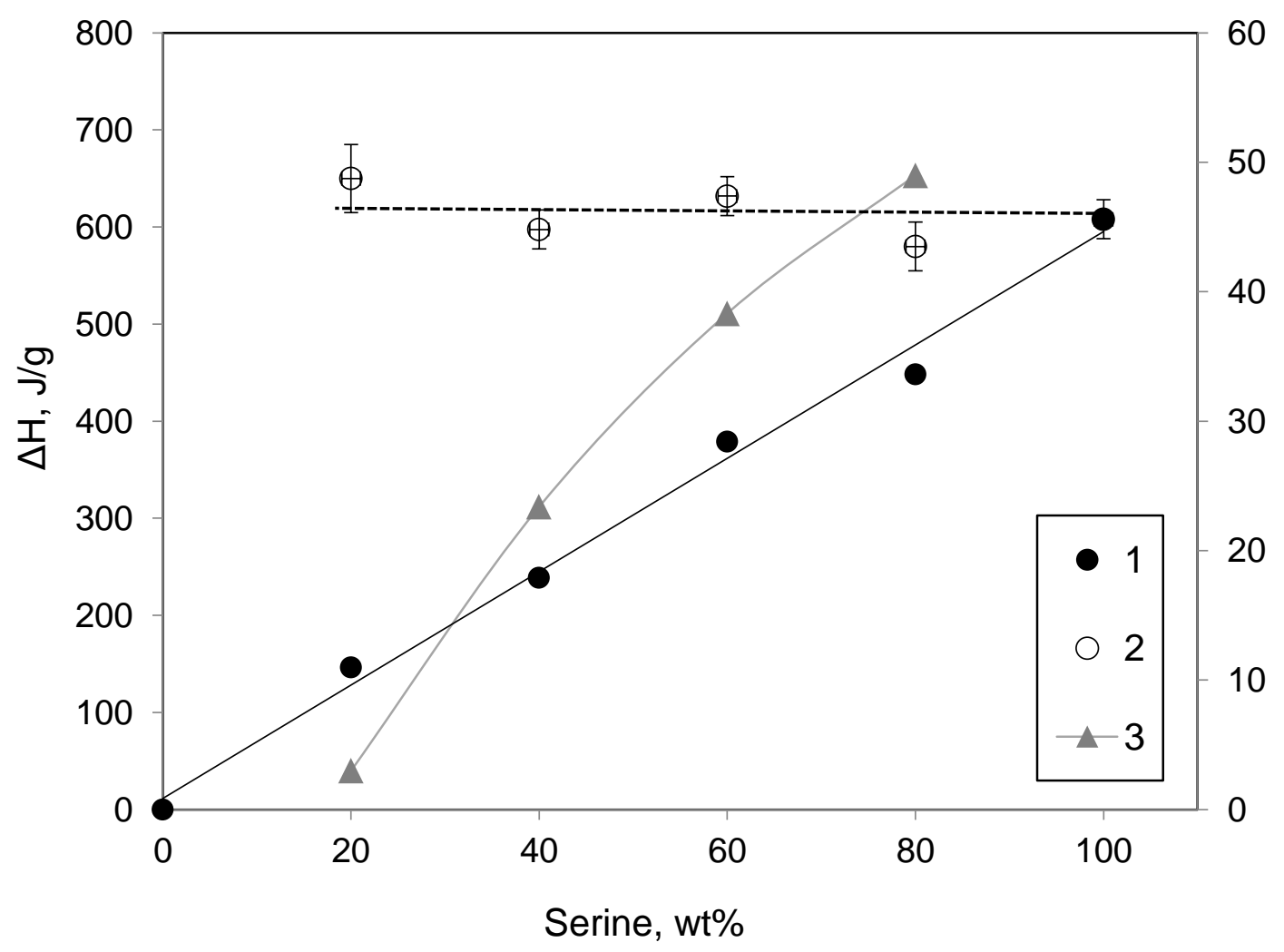

Fig. 7. Temperature behavior of lyophilized formulations studied by DSC.

(a): DSC thermograms of dry lyophilized samples: 
(1) $100 \%$ PVP30K;

(2) $100 \%$ mannitol;

(3) $100 \%$ serine;

(4) 2-excipient ODT (Man 20 wt\%, Ser 80 wt\%);

(5-7) 3-excipient ODTs comprising mannitol (20 wt\% fixed), Ser and PVP30K (5-7). Tablet compositions, wt\%: Ser 60, PVP 20 (5); Ser 40, PVP 40 (6); Ser 20, PVP 60 (7).

Samples were heated from -40 to $310^{\circ} \mathrm{C}$ at $20^{\circ} \mathrm{C} / \mathrm{min}$.

(b): Dependencies of the melting enthalpies, $\Delta \mathrm{H}$, for serine (1 (apparent values) and 2 (corrected)) and mannitol (3) on the serine concentration in the 3-excipient lyophilized ODTs containing mannitol (20 wt\% fixed), serine and PVP30K. Data 2 were re-calculated from the Ser melting peaks taking into account the actual concentration of serine in formulations.

Fig. 8.

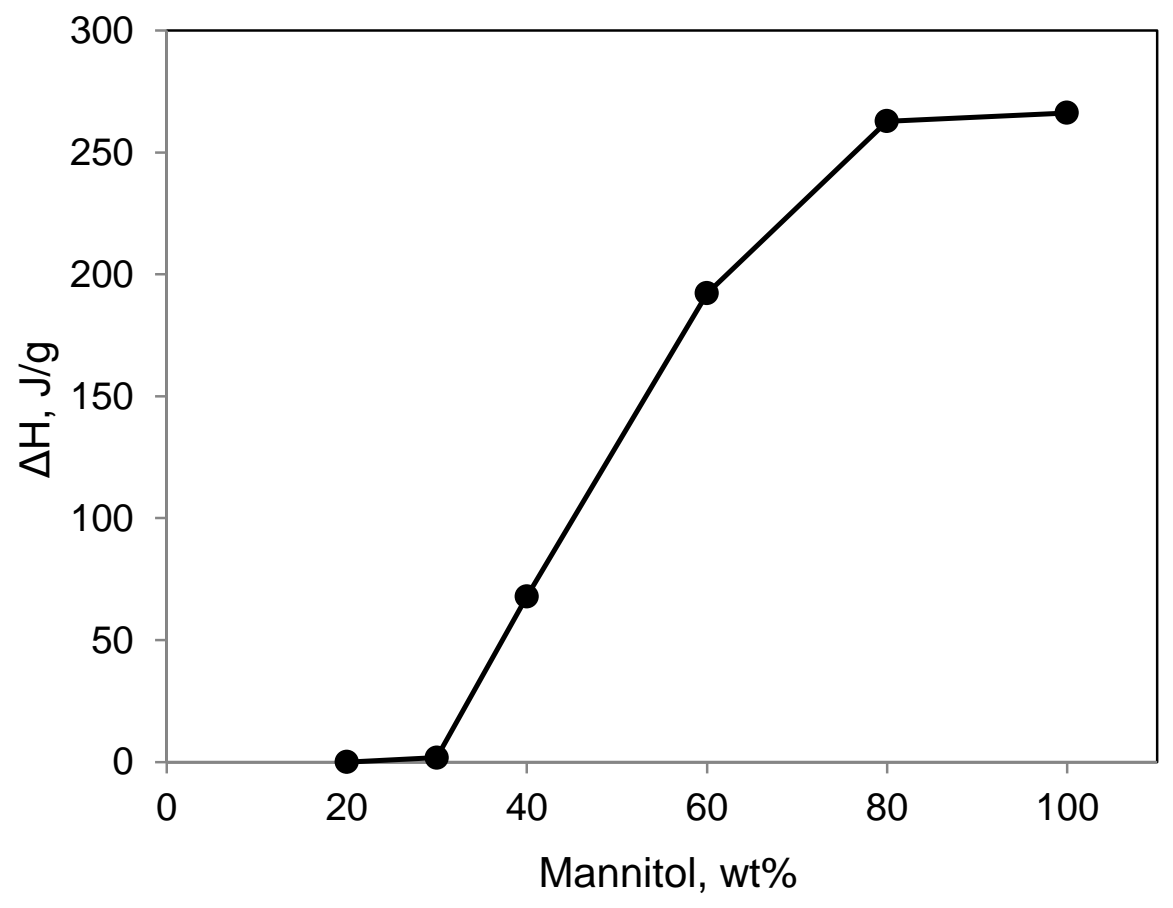

Fig. 8. Dependence of the mannitol melting enthalpy, $\Delta \mathrm{H}$, on the mannitol concentration in the 2-excipient lyophilized ODTs containing mannitol and PVP30K. All samples contain $10 \% \mathrm{w} / \mathrm{w}$ solid material. 


\section{References}

(1) World Health Organisation (WHO). Report of the informal expert meeting on dosage forms of medicines for children. WHO Headquarters, Geneva, Switzerland. 2008. http://www.who.int/selection_medicines/committees/expert/17/application/paediatric/Dosage_form_rep ortDEC2008.pdf Accessed 25 Jul 2018.

(2) Quinn HL, Hughes CM, Donnelly RF. Novel methods of drug administration for the treatment and care of older patients. Int J Pharm. 2016; 512(2): 366-73.

(3) Elwerfalli AM, Ghanchi Z, Rashid F, Alany RG, ElShaer A. New generation of orally disintegrating tablets for sustained drug release: A propitious outlook. Curr Drug Deliv. 2015; 12(6): 652-7.

(4) Stoltenberg I, Breitkreutz J. Orally disintegrating mini-tablets (ODMTs) - A novel solid oral dosage form for paediatric use. Eur J Pharm Biopharm. 2011; 78: 462-9.

( 5 ) AlHusban FA, El-Shaer AM, Jones RJ, Mohammed AR. Recent patents and trends in orally disintegrating tablets. Recent Pat Drug Deliv Formul. 2010; 4(3): 178-97.

(6) Lai M, Estrada M, Zhu C. Developing a Flexible Pediatric Dosage Form for Antiretroviral Therapy: A FastDissolving Tablet. J Pharm Sci. 2017; 106(8): 2173-7.

(7) Sastry SV, Nyshadham JR, Fix JA. Recent technological advances in oral drug delivery. A review. Pharm Sci Technol Today. 2000; 3(4): 138-45.

(8) Alyami H, Koner J, Huynh C, Terry D, Mohammed AR. Current opinions and recommendations of paediatric healthcare professionals - The importance of tablets: Emerging orally disintegrating versus traditional tablets. PLoS ONE. 2018; 13(2): e0193292.

(9) Courtney P, Alqurshi A, Forbes B, Royall PG, Strang J. Freeze-dried formulations: a new perspective on reformulating naloxone. Eur Pharm Rev. 2017; 3: 32-36.

(10) Lal M, Priddy S, Bourgeois L, Walker R, Pebley W, Brown J, Desai J, Darsley MJ, Kristensen D, Chen D. Development of a fast-dissolving tablet formulation of a live attenuated enterotoxigenic $\mathrm{E}$. coli vaccine candidate. Vaccine. 2013; 31(42): 4759-64.

(11) Wilkhu JS, McNeil SE, Anderson DE, Kirchmeier M, Perrie Y. Development of a solid dosage platform for the oral delivery of bilayer vesicles. Eur J Pharm Sci. 2017; 108: 71-7.

(12) Global Market Study on Orally Disintegrating Tablets: Anti-Psychotics Drug Class Segment to Record Market Attractiveness Index of 2.4 over the Forecast Period 2017 - 2025.2017. https://www.persistencemarketresearch.com/market-research/orally-disintegrating-tablet-market.asp.

Accessed $25 \mathrm{Jul} 2018$.

(13) Badgujar BP, Mundada AS. The technologies used for developing orally disintegrating tablets: a review. Acta Pharm. 2011; 61(2): 117-39.

(14) AlHusban F, Perrie Y, Mohammed AR. Formulation and characterisation of lyophilised rapid disintegrating tablets using amino acids as matrix forming agents. Eur J Pharm Biopharm. 2010; 75(2): 25462. 
(15) AlHusban FA, El-Shaer AM, Jones RJ, Mohammed AR. Recent patents and trends in orally disintegrating tablets. Recent Pat Drug Deliv Formul. 2010; 4(3): 178-97.

(16) Muir I. Growing sales and new opportunities for oral fast dissolve. In: Oral delivery: when you find the holy grail, on drug delivery. 2007. http://www.ondrugdelivery.com/publications/Oral_Drug_Delivery_07.pdf. Accessed 25 Jul 2018.

(17) Pikal MJ. Freeze Drying. Encyclopedia of pharmaceutical technology. 2002;1299-1326.

(18) Kasper JC, Winter G, Friess W. Recent advances and further challenges in lyophilization. Eur J Pharm Biopharm. 2013; 85(2): 162-9.

(19) Siew A. A Case for Orally Disintegrating Tablets. Pharm Tech. 2016;8:28-30.

(20) Parkash V, Maan S, Deepika, Yadav SK, Hemlata, Jogpal V. Fast disintegrating tablets: Opportunity in drug delivery system. J Adv Pharm Technol Res. 2011; 2(4): 223-35.

(21) Hackl EV, Ermolina I., Using Texture Analysis technique to assess the freeze-dried cakes in vials. J Pharm Sci. 2016; 105: 2073-85.

(22) Fu Y, Yang S, Jeong SH, Kimura S, Park K. Orally Fast Disintegrating Tablets: Developments, Technologies,T aste-Masking and Clinical Studies. Critical Reviews ${ }^{\mathrm{TM}}$ in Therapeutic Drug Carrier Systems. 2004; 21(6): 433-75.

(23) Segar H. Drug delivery products and the Zydis fast dissolving dosage. J Pharm Pharmacol. 1998; 50: 375-83.

(24) Chandrasekhar R, Hassan Z, Alhusban F, Smith AM, Mohammed AR. The role of formulation excipients in the development of lyophilised fast-disintegrating tablets. Eur J Pharm Biopharm. 2009; 72(1): 119-29.

(25) Ohrem HL, Schornick E, Kalivoda A, Ognibene R. Why is mannitol becoming more and more popular as a pharmaceutical excipient in solid dosage forms? Pharm Dev Technol. 2014; 19(3): 257-62.

(26) Le AS, Mulderrig KB. Sorbitol and Mannitol. In: Nabors LO, ed. Alternative Sweeteners, Third Edition. New York: Marcel Dekker, Inc; 2001. p. 317-334.

(27) $\mathrm{Wu} \mathrm{SH}$, Krieger DG, Park JH. An alternative to the USP disintegration test for orally disintegrating tablets. Pharm Technol. 2008; 32(8): 1-6.

http://www.pharmtech.com/alternative-usp-disintegration-test-orally-disintegrating-tablets. Accessed 25 Jul 2018.

(28) Orally Disintegrating Tablet and Film Technologies: Technologies, Market Analysis, \& Business Opportunities. In: Market Study Reports. 2013.

https://technology-catalysts.com/multi-client-report/orally-disintegrating-tablet-and-film-technologies-7thedition. Accessed 25 Jul 2018.

(29) Shallenberger RS. Taste Chemistry. Springer Science \& Business Media; 1993 
(30) Nair B. Final report on the safety of POLYVINYLPYRROLIDONE (PVP). Int J Toxicol. 1998; 17(Suppl. 4): 95 130.

(31) Patel SM, Nail SL, Pikal MJ, Geidobler R, Winter G, Hawe A, Davagnino J, Rambhatla Gupta S. Lyophilized drug product cake appearance: What is acceptable? J Pharm Sci. 2017; 106(7): 1706-21.

(32) Devi S, Williams DR. Density dependent mechanical properties and structures of a freeze dried biopharmaceutical excipientdsucrose. Eur J Pharm Biopharm. 2014; 88: 492-501.

(33) Devi S, Williams DR. Morphological and compressional mechanical properties of freeze dried mannitol, sucrose, and trehalose cakes. J Pharm Sci. 2013; 102: 4246-55.

(34) Hosford W. Mechanical behavior of materials. Cambridge: Cambridge University Press; 2005

(35) Callister WD, Rethwisch DG. Fundamentals of materials science and engineering: An integrated approach. 4th ed. New Jersey: John Wiley \& Sons; 2012

(36) Peleg M. On fundamental issues in texture evaluation and texturization. Food Hydrocolloids. 2006; 20 : 405-14.

(37) Peleg M. Review: mechanical properties of dry cellular solid foods. Food Sci Tech Int. 1997; 3: 227-40.

(38) Ullrich S, Seyferth S, Lee G. Measurement of shrinkage and cracking in lyophilized amorphous cakes. Part I: final-product assessment. J Pharm Sci. 2015; 104: 155-64.

(39) Hackl E, Darkwah J, Smith G, Ermolina I. Effect of Arginine on the Aggregation of Protein in Freeze-Dried Formulations Containing Sugars and Polyol: 1-Formulation Development. AAPS PharmSciTech. 2018; 19(2): 896-911.

(40) Hooper P, Lasher J, Alexander KS, Baki G. A new modified wetting test and an alternative disintegration test for orally disintegrating tablets. J Pharm Biomed Anal. 2016; 120: 391-6.

(41) Li B, Robinson JR. Preclinical assessment of oral mucosal drug delivery systems. In: Ghosh TK, Pfister WR, editors. Drugs and Pharmaceutical Sciences, Drug Delivery to the Oral Cavity: Molecules to Market. Boca Raton, Florida: CRC Press, Taylor \& Francis Group; 2005. p. 41-66.

(42) Food and Drug Administration, Center for Drug Evaluation and Research (FDA/CDER), Guidance for industry: orally disintegrating tablets, U.S. Department of Health and Human Services, U.S.A., 2008. https://www.fda.gov/downloads/Drugs/.../Guidances/ucm070578.pdf. Accessed 25 Jul 2018.

(43) McLaughlin R, Banbury S, Crowley K. Orally Disintegrating Tablets: The Effect of Recent FDA Guidance on ODT Technologies and Applications. Pharm Tech. 2009 Suppl; 5. http://www.pharmtech.com/orallydisintegrating-tablets-effect-recent-fda-guidance-odt-technologies-and-applications. Accessed $25 \mathrm{Jul} 2018$.

(44) Chatterjee K, Shalaev EY, Suryanarayanan R. Partially crystalline systems in lyophilization: I. Use of ternary state diagrams to determine extent of crystallization of bulking agent. J Pharm Sci. 2005; 94 (4): 798808. 
(45) Walkiria S. Schlindwein WS, Gibson M., editors. Pharmaceutical Quality by Design: A Practical Approach. Hoboken, NY: John Wiley and Sons; 2018.

(46) Van Bockstal PJ, De Meyer L, Corver J, Vervaet C, De Beer T. Noncontact infrared-mediated heat transfer during continuous freeze-drying of unit doses. J Pharm Sci. 2017; 106(1): 71-82.

(47) Corver J, Bockstal P-JV, De Beer T. A continuous and controlled pharmaceutical freeze-drying technology for unit doses. Eur Pharm Rev. 2017, 6.

https://www.europeanpharmaceuticalreview.com/article/70823/continuous-controlled-pharmaceuticalfreeze-drying-technology-unit-doses/2/\#p2. Accessed 25 Jul 2018. 